\title{
MORFOMETRÍA DE LAS CUENCAS DE LA RED HIDROGRÁFICA DE BIZKAIA (PAÍS VASCO, ESPAÑA)
}

\author{
L. Docampo', B.G. de Bikuña ${ }^{2}$, E. Rico' y A. Rallo' \\ 1. Laboratorio de Zoología, Departamento de Biología Animal y Genética, Facultad de Ciencias, Universidad \\ del País Vasco. E.H.U. Apdo 644, 48080 Bilbao. Spain. \\ 2. Laboratorio de Ecología, Departamento de Biología Vegetal y Ecología, Facultad de Ciencias, Universidad del \\ País Vasco. E.H.U. Apdo 644, 48080 Bilbao. Spain.
}

Palabras clave: river, basin, morphometry, allometric growth, drainage density, Spain.

\author{
ABSTRACT \\ MORPHOMETRY OF THE BASINS OF THE HYDROGRAPHIC NETWORK OF BISCAY \\ (BASQUE COUNTRY)
}

The morphometry of the 21 basins of the hydrographic network of Biscay (Spain) with 178 sampled sites has been studied. The shape of the basins is measured by the Gravelius and Caquot indices, the elongated, rounded forms predominating. The organization of the drainage system is demonstrated by the Schumm-Strahler method, and Horton's laws are tested. The study of the growth of the area by function of the mean drainage length is analysed by the allometric growth law. We put forward the use of the exponential growth law for the determination of the relative rates of the increasing area.

Biscay shows a not much dense hydrographic network for the most part $\left(0.65 \mathrm{~km} / \mathrm{km}^{2}\right)$, basically due both to the lithological characteristics and the thick plant cover in the rural areas which works as a barrier opposite to the potential erosion. The main part of the basins show a not much envolved erosion system excepting the Ibaizabal wich is the largest basin we have dealt with, the Galindo and in lesser extent the Cadagua, Butrón y Oka.

The influence of the lithological, topographical and climatic characteristics of the different basins is demonstrated by morphological analysis. Moreover, this study serves to interpret biotypological aspects and the behaviour of the rivers acting as ecosystems. At this point, a wide habitat heterogeneity is observed in the basins of Biscay.

\section{INTRODUCCIÓN}

Entre los trabajos realizados hasta el momento en la red fluvial de Bizkaia sobre medidas y análisis de la superficie, forma y dimensiones de las formas de relieve de las cuencas, están los llevados a cabo por Antigüedad \& Cruz-Sanjulián (1980) en la cuenca del río Arratia; CRUZ-SANJULIÁN \& SAENZ (1980) en la cuenca alta del río Nervión y ERASO \& ANTIGÜEDAD (1984) en las cuencas de los ríos Butrón, Oka, Lea y Artibai.

El análisis matemático de la configuración de la superficie, forma y dimensiones del relieve de las

Limnética, 5: 51-67 (1989)

(C) Asociación Española de Limnología, Madrid, Spain cuencas que constituyen la red hidrográfica de Bizkaia, permitirá que se puedan determinar, en posteriores trabajos, posibles relaciones con aspectos ecológicos. En este sentido, la cuenca o cualquier otra unidad hidrográfica, aunque comprenda ecosistemas distintos, forma en su conjunto una unidad ecológica de gran valor. Asimismo, los estudios hidromorfológicos realizados bajo un prisma ecológico enlazan con los aspectos de índole territorial y de ordenación, en especial a través del problema de la erosión (RAYNAL, 1970). Teniendo en cuenta el objetivo del presente trabajo, no se han aplicado algunos parámetros morfométricos-como curvas hipsométricas, rectángulo equivalente, índices de pendiente 
etc.- que se utilizan en hidrogeología, sino que se presenta una caracterización morfométrica de la red de drenaje de las 21 cuencas o subcuencas principales que constituyen la red hidrográfica de Bizkaia, en la cual se situaron 178 estaciones de muestreo. Entre los parámetros medidos, los relativos a las características de forma y el estudio del crecimiento alométrico han sido objeto de especial atención. Por último, se discuten las implicaciones biotipológicas en relación a la geomorfología de algunas cuencas, la cual ha sido tratada más específicamente en otros trabajos anteriormente citados.

\section{MATERIAL Y MÉTODOS}

La primera etapa de este estudio ha consistido en la elaboración de los mapas hidrológicos de cuencas. Para ello se han utilizado los mapas topográfico a escala 1:50000 del Servicio Geográfico del Ejército, sobre los que se ha realizado el conjunto de medidas. En el trazado de la red de drenaje de cada cuenca se han considerado únicamente los cursos de agua de trazado continuo, la mayoría de carácter permanente, y algunos temporales. Aunque con este método se obtienen valores de los parámetros morfométricos inferiores a los obtenidos con otros métodos, que consideran absolutamente todos los «talwegs», proporciona resultados suficientemente precisos.

Las medidas de longitud (segmentos y perímetros) se han realizado con un curvímetro convencional, calculándose las áreas mediante el método de la pesada, utilizando papel poliester, en balanza Mettler H10W con una precisión de $\pm 0,1 \mathrm{mg}$, lo cual equivale a $\pm 1 \times 10^{-11} \mathrm{~km}^{2}$. Este método no ofrece diferencias apreciables respecto del cálculo de las áreas mediante planimetría, como ha sido demostrado por ANTIGüEDAD \& CRUz-SANJULián (op. cit.). En nuestro caso hemos podido comprobar que la suma de las áreas de todas las cuencas estudiadas es de $2740,74 \mathrm{~km}^{2}$ - dentro de la cual están incluidas algunas zonas de las provincias de Santander, Burgos y Álava - frente a los $2217 \mathrm{~km}^{2}$ que tiene el área política de Bizkaia.

Los índices morfométricos empleados son el coeficiente de Gravelius, alargamiento medio de Caquot, área media parcial de cada estación de muestreo, constante de alometría, densidad de drenaje, constante de mantenimiento del canal y la frecuencia de los cauces (Roche, 1963; REMENIERAS, 1971).

\section{RESULTADOS}

\section{Características de forma}

Las características de forma de las cuencas se resumen en aquellos parámetros que contemplan la forma de las mismas, hecha abstracción de su relieve. Para la aplicación de los índices de forma de Gravelius y de Caquot, debe determinarse el perímetro estilizado de cada cuenca, $\mathrm{P}$, despreciando sinuosidades no significativas; su área, $\mathrm{S}$,

Tabla 1.- Valores de los diferentes parámetros que determinan la forma de las cuencas de la red fluvial de Bizkaia. S: área de la cuenca $\left(\mathrm{km}^{2}\right)$; L: longitud total de drenaje $(\mathrm{km})$; W: razón de elongación; Kc: índice de compacidad de Gravelius; $\mathrm{Ca}$ : alargamiento medio de Caquot; $\mathbf{O}$ : orientación del eje principal; N: número de estaciones muestreadas.

Values of the different form parameters of Biscay's hydrographic basins. S: area of the basin $\left(\mathrm{km}^{2}\right)$; L: drainage length $(\mathrm{km})$; W: elongation ratio; Kc: Gravelius index; Ca: Caquot index; O: orientation of the principal river; $\mathrm{N}$ : sampled sites number.

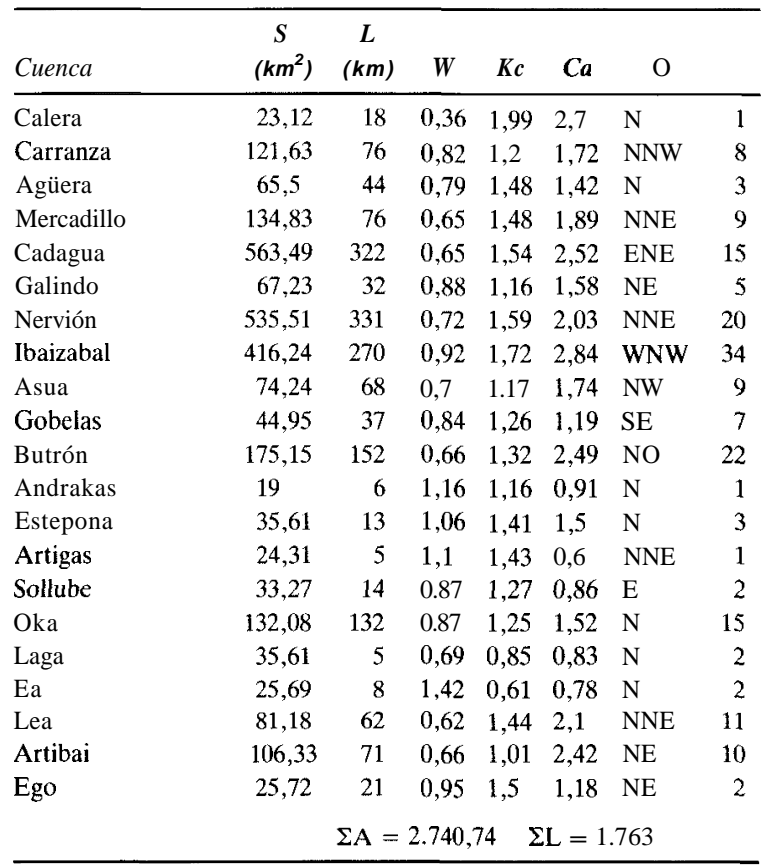


y el máximo recorrido entre la periferia y la salida de la cuenca, E.

a) Índice de compacidad de Gravelius (Kc)

Este índice indica la circularidad de la cuenca, y se calcula como la relación entre el perímetro de la cuenca y el de un círculo que tuviera su misma superficie:

$$
\mathrm{Kc}=\frac{\mathrm{P}}{2 \sqrt{\pi S}}
$$

Así pues, si $\mathrm{Kc}=1$ la cuenca es circular, tomando un valor de 1,157 en una cuenca semicircular. Este valor será tanto mayor cuanto más alargada sea la cuenca. Los valores Kc obtenidos para las cuencas vizcaínas se dan en la tabla 1 . b) Alargamiento medio de Caquot $(\mathrm{Ca})$

Este índice define la relación entre el recorrido más largo desde la periferia a la salida de la cuen- ca con respecto a la longitud del lado de un cuadrado de área equivalente:

$$
\mathrm{Ca}=\frac{\mathrm{E}}{\sqrt{\mathrm{S}}}
$$

Si $\mathrm{Ca}=\sqrt{2}$, la cuenca es cuadrada. Tomaría un valor de 1,128 en una cuenca circular; de 1,596 en una cuenca semicircular con la salida en un extremo del diámetro; y de 0,798 en una cuenca semicircular con la salida en el centro del diámetro. Como en el caso anterior, las cuencas más alargadas presentan un coeficiente de Caquot más elevado. En la tabla 1 se han señalado también los valores de Ca para las cuencas estudiadas.

El grado de asociación (correlación de Pearson r), entre Kc y Ca para las cuencas de Bizkaia es de 0,56 (g.l. = 19; p < 0,01), lo cual implica que el $68 \%\left(1-r^{2}\right)$ de la variabilidad del índice de Gra-

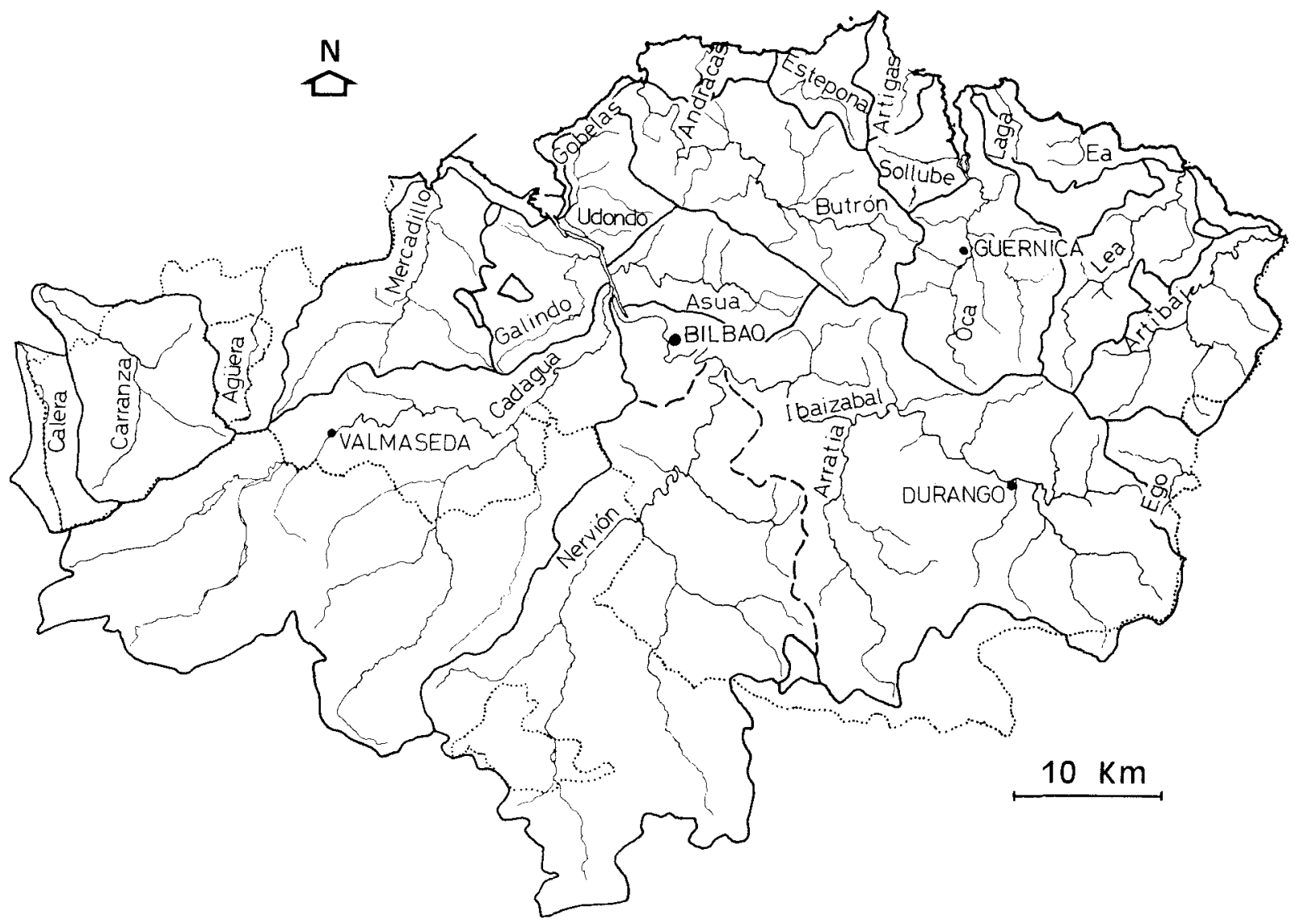

Figura 1.- Área de estudio (se indican solamente los cursos más importantes) Study area (only the most important rivers are indicated). 
velius no es explicable por la variación del índice de Caquot. La norma es que los coeficientes Kc y Ca estén correlacionados de forma directamente proporcional; es decir, que los valores de circularidad corroboren los resultados obtenidos con la constante de alargamiento de la cuenca. Esto se cumple en las cuencas que tienen una forma regular, pero no así en las irregulares, en que puede suceder que $\mathrm{Kc}$ indique circularidad y $\mathrm{Ca}$ alargamiento o viceversa. Así, tenemos que el $32 \%$ del ajuste de la varianza de Kc es atribuible a la forma regular que presentan 12 cuencas: Calera, Agüera, Mercadillo, Cadagua, Nervión, Ibaizabal, Gobelas, Butrón, Andrakas, Sollube, Oka y Lea. Dentro de este grupo, las que tienen forma circular son Gobelas, Andrakas y Sollube; cuencas de forma muy alargadas son Calera, Mercadillo, Cadagua, Nervión, Ibaizabal, Butrón y Lea; la cuenca que presenta una forma más cuadrada es la del Agüera, siendo la del Oka de forma sermicircular con salida en un extremo del diámetro.

El resto de las cuencas presentan una superficie bastante irregular debido a ensanchamientos en diferentes zonas, normalmente en las cabeceras y en las desembocaduras. A este respecto cabe destacar la cuenca del Galindo, que tiene una forma circular con un ensanchamiento originado por el afluente Ballonti y con salida en el extremo del diámetro. El Asúa, cuenca alargada, con un engrosamiento en su zona central, tiene una forma aproximadamente rectangular. La cuenca del Artibai, de forma circular, presenta un fuerte estrechamiento en la desembocadura, y las cuencas costeras como Sollube, Artigas, Laga y Ea, con un recorrido comprendido entre 3 y $9 \mathrm{~km}$ y que atraviesan un relieve de pendientes pronunciadas, son de forma semicircular, con su salida en el centro del diámetro $(0,6 \mathrm{a} \mathrm{Ca} \leqslant 0,86)$. El Estepona, de características similares a los anteriores, es moderadamente semicircular, con un alargamiento originado por la trayectoria del eje principal. Todas estas deformaciones se deben a las alometrías que manifiesta el crecimiento relativo del área en función de la longitud de drenaje.

\section{Jerarquización de las cuencas}

Con los métodos propuestos por STRAHLER (1952) y Sснumm (1956), y utilizando los cursos

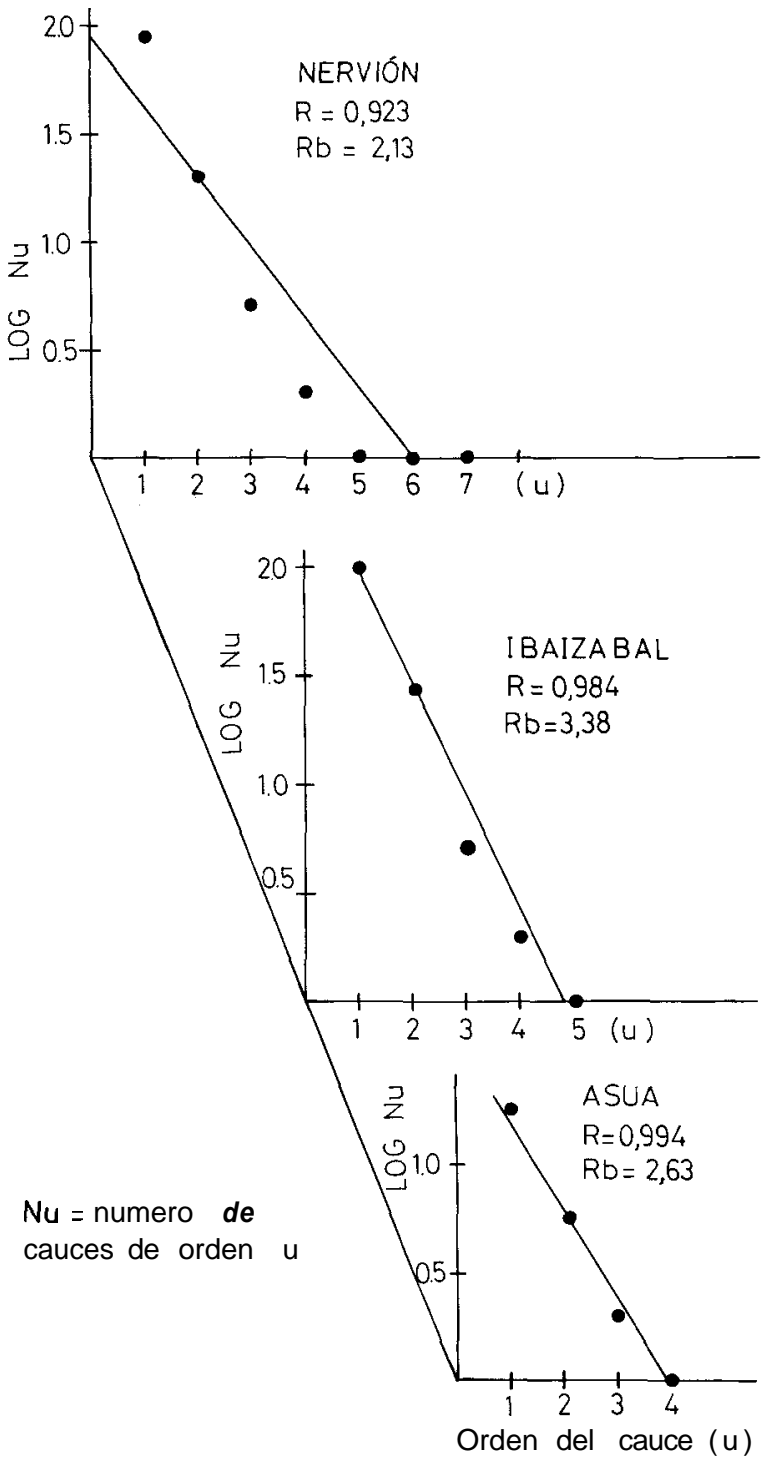

Figura 2.- Determinación de la razón de bifurcación media $(\mathrm{Rb})$ en las cuencas de los ríos Nervión, Ibaizabal y Asua. Nu: número de cauces de orden u; r: coeficiente de correlación de Pearson.

Determination of the mean fork ratio $(\mathrm{Rb})$ in the basins of the rivers Nervion. Ibaizabal and Asua. Nu: number of streams of degree u; r: Pearson's correlation coefficient.

de agua de carácter permanente ası como los estacionales de recorrdio largo, se ha efectuado la jerarquización completa de todas las cuencas de la red hidrográfica de Bizkaia, que se muestran en la fig. 1. 
La ley del número de los cauces de HorTon (1945) establece que la relación de bifurcación $(\mathrm{Rb}=\mathrm{Nu} / \mathrm{Nu}+1$, igual al número de cauces de orden u dividido por el número de cauces de orden inmediatamente superior) tiende a permanecer constante, siendo la razón de una progresión geométrica inversa a que da lugar el número total de segmentos de órdenes sucesivos (fig. 2):

$$
\begin{gathered}
\mathrm{Nu}=\alpha R b^{u} \\
\log \mathrm{Nu}=\log \mathrm{a}+\mathrm{u} \log \mathrm{Rb}
\end{gathered}
$$

A partir de la recta de regresión semilogarítmica entre $\log \mathrm{Nu}$ y u se determina $\mathrm{Rb}$, que es pre- cisamente el antilogaritmo de la pendiente de dicha recta. De esta forma se obtiene un valor de la relación de bifurcación representativo del conjunto de la cuenca. En la tabla 2 se presentan los valores de $\mathrm{Rb}$ entre los distintos órdenes para cada una de las cuencas estudiadas, así como el valor medio y la desviación típica $(\overline{\mathrm{Rb}} \pm \mathrm{a}) \mathrm{de}$ cada una de ellas. En la tabla 3 se señalan los valores de $\mathrm{Rb}$ obtenidos mediante la ecuación (4). En las cuencas que presentan una correlación y regresión lineal estadísticamente significativa, los valores de $\mathrm{Rb}$ son similares a los valores medios obtenidos de forma empírica (tabla 2).

Tabla 2.- Ordenes $(\mathrm{U})$, número de segmentos $(\mathrm{Nu})$ de cada orden y radio de bifurcación $(\mathrm{Rb})$ de las cuencas de los ríos estudiados. Aplicación de la jerarquización de Schumm-Strahler.

Orders $(\mathrm{U})$, number of riverbeds of each order $(\mathrm{Nu})$ and bifurcation ratio $(\mathrm{Rb})$ of the rivers studied. Application of the Schumm-

\begin{tabular}{|c|c|c|c|c|c|c|c|c|c|}
\hline Cuenca & $U$ & $N u$ & $R b$ & $R b \pm o$ & Cuenca & $U$ & $N u$ & $R b$ & $R b \pm 0$ \\
\hline \multirow[t]{2}{*}{ Calera } & 1 & 4 & & & Asua & 1 & 18 & & \\
\hline & 2 & 1 & 4 & $4 \pm 0$ & & 2 & 6 & 3 & \\
\hline \multirow[t]{3}{*}{ Carranza } & 1 & 20 & & & & 3 & 2 & 3 & \\
\hline & 2 & 5 & 4 & & & 4 & 1 & 2 & $2,66 \pm 0.57$ \\
\hline & 3 & 1 & 5 & $4,5 \pm 0,70$ & Butrón & 1 & 40 & & \\
\hline \multirow[t]{3}{*}{ Agüera } & 1 & 15 & & & & 2 & 10 & 4 & \\
\hline & 2 & 2 & 7,5 & & & 3 & 2 & 5 & \\
\hline & 3 & 1 & 2 & $4,75 \pm 3,88$ & & 4 & 1 & 2 & $3,66 \pm 1,52$ \\
\hline \multirow[t]{3}{*}{ Mercadillo } & 1 & 18 & & & Andrakas & 1 & 2 & & \\
\hline & 2 & 6 & 3 & & & 2 & 1 & 2 & $2 \pm 0$ \\
\hline & 3 & 1 & 6 & $4,5 \pm 2,12$ & Estepona & 1 & 4 & & \\
\hline \multirow[t]{5}{*}{ Cadagua } & 1 & 109 & & & & 2 & 1 & 4 & $4 \pm 0$ \\
\hline & 2 & 15 & 7,26 & & Sollube & 1 & 3 & & \\
\hline & 3 & 6 & 2,5 & & & 2 & 1 & 3 & $3 \pm 0$ \\
\hline & 4 & 2 & 3 & & Artigas & 1 & 2 & & \\
\hline & 5 & 1 & 2 & $3,69 \pm 2,41$ & & 2 & 1 & 2 & $2 \pm 0$ \\
\hline \multirow[t]{7}{*}{ Nervión } & 1 & 96 & & & Laga & 1 & 1 & - & - \\
\hline & 2 & 22 & 4,36 & & Oka & 1 & 35 & & \\
\hline & 3 & 5 & 4,4 & & & 2 & 8 & 4,37 & \\
\hline & 4 & 2 & 2,5 & & & 3 & 1 & 8 & $6,18 \pm 2,56$ \\
\hline & 5 & 1 & 2 & & $\mathrm{Ea}$ & 1 & 3 & & \\
\hline & 6 & 1 & 1 & & & 2 & 1 & 3 & $3 \pm 0,00$ \\
\hline & 7 & 1 & 1 & $2,54 \pm 1,53$ & Lea & 1 & 22 & & \\
\hline \multirow[t]{5}{*}{ Ibaizabal } & 1 & 124 & & & & 2 & 5 & 4,4 & \\
\hline & 2 & 29 & 4,27 & & & 3 & 2 & 2,5 & \\
\hline & 3 & 5 & 5,8 & & & 4 & 1 & 2 & $2,96 \pm 1,26$ \\
\hline & 4 & 2 & 2,5 & & Artibai & 1 & 16 & & \\
\hline & 5 & 1 & 2 & $3,64 \pm 1,73$ & & 2 & 5 & 3.2 & \\
\hline \multirow[t]{3}{*}{ Galindo } & 1 & 8 & & & & 3 & 2 & 2,5 & \\
\hline & 2 & 4 & 2 & & & 4 & 1 & 2 & $2,56 \pm 0,60$ \\
\hline & 3 & 1 & 4 & $\pm 1,41$ & Ego & 1 & 6 & & \\
\hline \multirow[t]{3}{*}{ Gobelas } & 1 & 8 & & & & 2 & 3 & 2 & \\
\hline & 2 & 2 & 4 & & & 3 & 1 & 3 & $2.50 \pm 0.70$ \\
\hline & 3 & 1 & 2 & $3 \pm 1,41$ & & & & & \\
\hline
\end{tabular}
Strahler's hierarchization. 
$\mathrm{Rb}$, normalmente, toma valores entre 3 y 4 (Hynes, 1970) o entre 3 y 5 (STrahler, 1977). Valores de $\mathrm{Rb}>4$ son indicadores de torrencialidad moderadamente alta; en este sentido las cuencas más torrenciales de Bizkaia son las de los ríos Carranza, Mercadillo y Oka, y las menos torrenciales, las del Galindo, Gobelas, Asua y Nervión. De este último grupo, las tres primeras son cuencas costeras cuya altitud máxima no sobrepasa los 100 m s.n.m. En el caso del Nervión, el eje principal, a partir de Delika, atraviesa tramos de altitud inferior a 300 m s.n.m., con pendientes en torno al $1 \%$.

La ley del área de las cuencas de Horton (op.cit.) determina que las áreas medias de las cuencas drenadas por segmentos de cauce de Órdenes crecientes sigan también una progresión geométrica, cuya razón es la relación de áreas $\left(\mathrm{R}_{\mathrm{A}}\right)$ :

$$
\begin{gathered}
\bar{A} \mathbf{u}+\bar{A} 1 R_{A}^{(u-1)} \\
\log \bar{A} u=\log \bar{A} 1+(u-1) \log R_{A}
\end{gathered}
$$

(siendo Au el área media hasta los segmentos de orden u, y $\bar{A} 1$, el área media de los de primer orden).

De modo idéntico a la ecuación (4), $R_{A}$ se puede determinar mediante el antilogaritmo de la

Tabla 3.- Determinación mediante la ley de Horton (ley del número de los cauces) de la relación de bifurcación (Rb) representativa del conjunto de la cuenca. Aplicación de la recta de regresión $\log \mathrm{Nu}=\log \mathrm{Z}+\log (\mathrm{Rb})$ (um-u). La correlación entre $\log \mathrm{Nu}$ y u no es significativa al $95 \%$; sin embargo, el coeficiente de regresión es significativo entre el $95 \%$ y

\begin{tabular}{|c|c|c|c|c|c|c|}
\hline Cuenca & $\begin{array}{l}\text { Ecuación de } \\
\text { regresión }\end{array}$ & $r$ & $t$ & g.l. & $p$ & $R b$ \\
\hline Carranza & $1,96-0,65 u$ & 0,999 & 65,25 & 1 & $<0,05$ & 4,46 \\
\hline Agüera & $1,66-0,58 \mathrm{u}$ & 0,962 & 10,3 & 1 & $>0,1$ & NS \\
\hline Mercadillo & $1,92-0,62 u$ & 0,989 & 20,24 & 1 & $*$ & 4,16 \\
\hline Cadagua & $2,33-0,49 u$ & 0,981 & 22,8 & 3 & $<0,005$ & 3,09 \\
\hline Nervión & $1,94-0,33 \mathbf{u}$ & 0,923 & 14,14 & 5 & $<0,005$ & 2,13 \\
\hline Ibaizabal & $2,51-0,53 u$ & 0,984 & 27,32 & 3 & $<0,005$ & 3,38 \\
\hline Galindo & $1,40-0,45 u$ & 0,981 & 15,05 & 1 & * & 2,81 \\
\hline Gobelas & $1,30-0,45 u$ & 0,981 & 15,05 & 1 & $*$ & 2,81 \\
\hline Asua & $1,64-0,42 u$ & 0,994 & 41,19 & 2 & $<0,01$ & 2,63 \\
\hline Butrón & $2,10-0,54 \mathrm{u}$ & 0,988 & 26,94 & 2 & $<0,02$ & 3,49 \\
\hline Oka & $2,35-0,77 \mathbf{u}$ & 0,995 & 29,73 & 1 & $\sim 0,05$ & 5,88 \\
\hline Lea & $1,69-0,44 u$ & 0,985 & 22,19 & 2 & $<0,02$ & 2,75 \\
\hline Artibai & $1,55-0,40 \mathrm{u}$ & 0,999 & 35,77 & 2 & $<0,01$ & 2,51 \\
\hline Ego & $1,18-0,38 \mathrm{u}$ & 0,991 & 22,73 & 1 & * & 2,39 \\
\hline
\end{tabular}
el $98 \%$.
Tabla 4.- Determinación de la relación de incremento de área constante $\left(R_{A}\right)$ mediante la ecuación $\log$. $A=\log$. $A,+u$ $\log \mathrm{r}_{\mathrm{A}}$. Se dan los valores del coeficiente de correlación de Pearson (r) y la t de Student para comprobar la significación del coeficiente de regresión.

Determination of the area increase ratio $\left(R_{A}\right)$ by equation $\log$ $\mathrm{A}=\log \overrightarrow{\mathrm{A}}_{1}+\mathrm{u} \log \mathrm{R}_{\mathrm{A}}$. The values of Pearson's correlation coefficient and Student's $t$ are given for testing the significance of the regression coefficient $\left(R_{A}\right)$.

\begin{tabular}{lcccccc}
\hline \multicolumn{1}{c}{$\begin{array}{c}\text { Ecuación de } \\
\text { regresión }\end{array}$} & $\boldsymbol{r}$ & $\boldsymbol{t}$ & $\boldsymbol{g} . \boldsymbol{l}$. & $\boldsymbol{p}$ & $\boldsymbol{R} \boldsymbol{b}$ \\
\hline Carranza & $-0,20+0,56 \mathbf{u}$ & 0,856 & 16,26 & 3 & $>0,05$ & NS \\
Agüera & $0,32+0,43 \mathbf{u}$ & 0,922 & 19,43 & 1 & $>0,05$ & NS \\
Mercadillo & $-0,35+0,75 \mathbf{u}$ & 0,883 & 15,09 & 7 & $<0,005$ & 5,62 \\
Cadagua & $0,17+0,53 \mathbf{u}$ & 0,95 & 30,01 & 13 & $<0,001$ & 3,38 \\
Nervión & $0,48+0,36 \mathbf{u}$ & 0,888 & 18,46 & 17 & $<0,001$ & 2,29 \\
Ibaizabal & $0,058+0,52 \mathbf{u}$ & 0,836 & 19,57 & 30 & $<0,001$ & 3,31 \\
Galindo & $-1,22+1,23 \mathbf{u}$ & 0,964 & 17,17 & 2 & $<0,05$ & 16,98 \\
Gobelas & $-0,49+0,63 \mathbf{u}$ & 0,973 & 27,14 & 5 & $<0,001$ & 4,26 \\
Asua & $-0,46+0,44 \mathbf{u}$ & 0,906 & 9,5 & 6 & $<0,005$ & 2,75 \\
Butrón & $-0,65+0,81 \mathbf{u}$ & 0,909 & 20,57 & 18 & $<0,001$ & 6,45 \\
Oka & $-0,14+0,58 \mathbf{u}$ & 0,678 & 11,94 & 13 & $<0,01$ & 3,8 \\
Lea & $0,18+0,25 \mathbf{u}$ & 0,428 & 3,64 & 9 & $<0,1$ & NS \\
Artibai & $0,40+0,30 \mathbf{u}$ & 0,847 & 12,09 & 8 & $<0,005$ & 1,99 \\
\hline
\end{tabular}

pendiente de la recta de regresión (6). La regresión se ha realizado entre el logaritmo del área parcial de cada estación de muestreo con el correspondiente orden (u) del cauce (tabla 4).

En el estudio realizado en la cuenca alta del río Nervión (Cruz-SANJulián \& S AENZ op.ct.), el valor $R_{A}$ obtenido varía entre 3,73 y 3,96 , considerados como valores normales. En el presente trabajo, en el que se manejan cuencas completas, los valores más altos de la razón constante de incremento del área lo presentan las cuencas de los ríos Butrón y Mercadillo. La cuenca del Galindo obtiene, asimismo, un valor muy elevado de $R_{A}$ debido a que el eje principal nace en la vertiente sur (orientación NE), mientras que su afluente principal nace muy alejado del mismo, cerca de la costa, lo que se traduce en un gran incremento del área al pasar a un orden de cauce inmediatamente superior (la cuenca tiende a tener la forma de la sección longitudinal de un ovoide).

\section{Ley del crecimiento relativo}

La cuenca, como sistema de erosión fluvial, tiene un crecimiento relativo que se puede aseme- 
jar al que presenta una planta o un animal poiquilotermo (STRAHLER, 1977). En su fase juvenil, la red de drenaje abre nuevas ramas o cursos en las partes elevadas; con el tiempo y con el ciclo de erosión se van formando nuevos cauces de primer orden y los más antiguos pasan a tener valores más elevados. Para determinar este crecimiento, los geomorfólogos aplican el modelo biológico del crecimiento relativo (ley de Huxley), que nosotros hemos podido ensayar en estudios biométricos (Docampo et al, 1987). Esta ley se basa en que si un cambio de tamaño no va acompañado de un cambio de forma, se da una relación sencilla entre sus dimensiones lineales, áreas y volúmenes equivalentes. Las dimensiones lineales crecerán según la ecuación de una recta, las áreas como el cuadrado, y los volúmenes como el cubo de la longitud. El modelo viene expresado por una ecuación sencilla de tipo potencial:

$$
\mathrm{y}=\mathrm{ax} \mathrm{x}^{\mathrm{b}}
$$

donde y representa la variable del órgano cuya velocidad relativa de crecimiento se trata de determinar en función de una medida patrón x representativa del desarrollo de todo el individuo (tamaño o peso). La aplicación de este modelo a la morfometría de una cuenca nos lleva a la expresión:

$$
\begin{gathered}
\bar{A} u=a \bar{L}^{b} \\
\text { 1n } \bar{A} u=\ln a+b \ln \bar{L} u
\end{gathered}
$$

El área parcial o media de una cuenca (Au) es proporcional a la longitud de drenaje media ( $\overline{\mathrm{L} u})$ que la drena. Dimensionando la ecuación (8), tenemos que:

$$
\begin{gathered}
\overline{\mathrm{A} u}=\mathrm{a} \overline{\mathrm{L}} \mathrm{u}^{\mathrm{b}} \\
|\overline{\mathrm{L}} \mathrm{u} \overline{\mathrm{H}}| \propto{\mathrm{a} \overline{\mathrm{L}} \mathrm{u}^{\mathrm{b}}}_{\overline{\mathrm{H}} \propto \mathrm{a} \overline{\mathrm{L}} \mathrm{u}^{\mathrm{b}-1}} \\
\overline{\mathrm{H}}=\mathrm{K} \overline{\mathrm{L}} \mathrm{u}^{\mathrm{b}-1}
\end{gathered}
$$

$\overline{\mathrm{H}}$ puede relacionarse con el concepto de $\sim$ anchura media» de la cuenca e indicará la relación existente entre Au y $\overline{\mathrm{L}} \mathrm{u}(\mathrm{H} \propto \overline{\mathrm{A}} \mathrm{u} / \overline{\mathrm{L}} \mathrm{u})$.

Según la ecuación (10), el crecimiento relativo de una cuenca se analiza del siguiente modo: a) $\mathrm{Si} b=2$, el crecimiento es isométrico y la relación $\overline{\mathrm{A}} \mathrm{u} / \overline{\mathrm{L}} \mathrm{u}$ crece linealmente $(\mathrm{H}=\mathrm{K} \overline{\mathrm{Lu}})$; el
Tabla 5.- Valores del coeficiente mórfico o constante de alometría (b), de la ordenada en el origen (In a) y del coeficiente de correlación de Pearson (r) entre el área parcial de cada estación, según el orden del río (Au), y la longitud acumulativa de la red de drenaje (Lu):

$\ln \mathrm{Au}=\ln \mathrm{a}+\mathrm{b} \ln \mathrm{L} u . u$

Values of the allometric constant (b), the ordinate in the origin (In a) and Pearson's correlation coefficient (r) between the partical area of each sampled site according to river's degree and the accumulative length of the drainage network (Lu): $\ln \mathrm{Au}=\ln \mathrm{a}+\mathrm{b} \ln \overline{\mathrm{L}} \mathrm{u}$

\begin{tabular}{lrrrrl}
\hline Cuenca & $b$ & \multicolumn{1}{c}{$\ln a$} & $r$ & $n$ & \multicolumn{1}{c}{$p$} \\
\hline Mercadillo & 1,307 & 0,411 & 0,928 & 9 & $<0,001$ \\
Cadagua & 1,458 & 0,343 & 0,97 & 15 & $<0,001$ \\
Galindo & 1,68 & $-0,503$ & 0,983 & 4 & $<0,02$ \\
Nervión & 1,376 & 0,748 & 0,952 & 19 & $<0,001$ \\
Ibaizabal & 1,638 & $-0,064$ & 0,972 & 33 & $<0,001$ \\
Asua & 1,252 & 0,119 & 0,863 & 8 & $<0,01$ \\
Gobelas & 1,353 & 0,014 & 0,912 & 7 & $<0,005$ \\
Butrón & 1,447 & $-0,019$ & 0,961 & 20 & $<0,001$ \\
Oka & 1,413 & 0,227 & 0,924 & 15 & $<0,001$ \\
Lea & 1,387 & $-0,266$ & 0,861 & 11 & $<0,001$ \\
Artibai & 0,932 & 0,831 & 0,655 & 10 & $<0,05$ \\
\hline
\end{tabular}

área crecería como el cuadrado de la longitud y la cuenca no experimentaría deformaciones durante su desarrollo. b) Cuando $b>2$, el crecimiento es alométrico positivo; Āu/Ľu crecería desproporcionadamente rápido respecto al incremento de ¿u. c) Si b $<2$, la alometría es negativa y Āu/ĹLu aumentaría muy poco respecto del aumento de $\overline{\mathrm{L}}$; generalmente, las alometrías de las cuencas son negativas, lo cual ocasiona un tipo de deformación muy corriente: a medida que aumenta la longitud de drenaje, las cuencas se hacen más estrechas y alargadas hasta acabar cerrándose.

Dos casos particulares de la ecuación (10) son aquellos en los que b a $\mathbf{1}$. En el primer caso, si $\mathrm{b}=1$, el área crece de forma lineal y $\mathrm{H}$ permanece constante $(\mathrm{H}=\mathrm{K})$. En este caso la deformación suele tener lugar en las primeras fases del crecimiento y la cuenca adquiere la figura aproximada de un rectángulo; después, el sistema continúa creciendo con la misma forma. PRAT et al. (1982), en la subcuenca del tío Ripoll (Llobregat), determinan un crecimiento de este tipo $(\mathrm{b}=1,135)$; SABATER (1987), obtiene la ecuación $\overline{\mathrm{L}} \mathrm{u}=0,12 \overline{\mathrm{Au}} \mathrm{u}^{0,92}$, de donde se puede calcular que $\mathrm{b}=1,086$ en la cuenca del río Ter, indicando que el crecimiento es prácticamente lineal, ya que se trata de una cuenca de forma alargada y unifor- 
me, que puede descomponerse en rectángulos. En el segundo caso, cuando $\mathrm{b}<1\left(\mathrm{H}=\mathrm{K} \mathrm{Lu}^{-2}\right.$, siendo $\mathrm{z}<1$ ), $\mathrm{H}$ disminuye al incrementarse la longitud, lo cual suele ser resultado de una deformación brusca en una zona concreta de la cuenca.

La constante a indica siempre la superficie que tiene una cuenca cuya longitud de drenaje es de $1 \mathrm{~km}$. De esta forma, cuanto menor sea la alometría negativa de una cuenca (cuanto más se aproxime $\mathrm{b}$ al valor 2), más madura será, lo que implica que su sistema de erosión está más avanzado. Geológica y biológicamente, no es viable un sistema donde $b \leqslant 0$, y tampoco se han obtenido valores de $b \geqslant 2$. Los valores más altos de b deben encontrarse en torno a 1,80-1,90; JIMÉNEZ \& MARTíneZ-LOPEZ (1988), en un estudio del río Júcar, obtienen un valor de $b=1,851$. PalaU (1987), considerando constante el valor de a calculado por otros autores en otras cuencas, obtiene una alometría de 1,86 para la cuenca del río Segre. Sin embargo, y de acuerdo con lo expuesto por Margalef (1974), la ecuación de crecimiento es característica de la población, pero no de la especie. Es por ello que el cálculo de las constantes a y b debe realizarse a partir de las propias mediciones de áreas medias y longitudes, admitiendo comparaciones pero no extrapolaciones.

En la tabla 5 están expresadas las alometrías de las principales cuencas de Bizkaia. Las que se encuentran en un estado más maduro son las del Galindo e Ibaizabal, donde se ha obtenido un valor de $b$ similar al obtenido por otros autores en otras

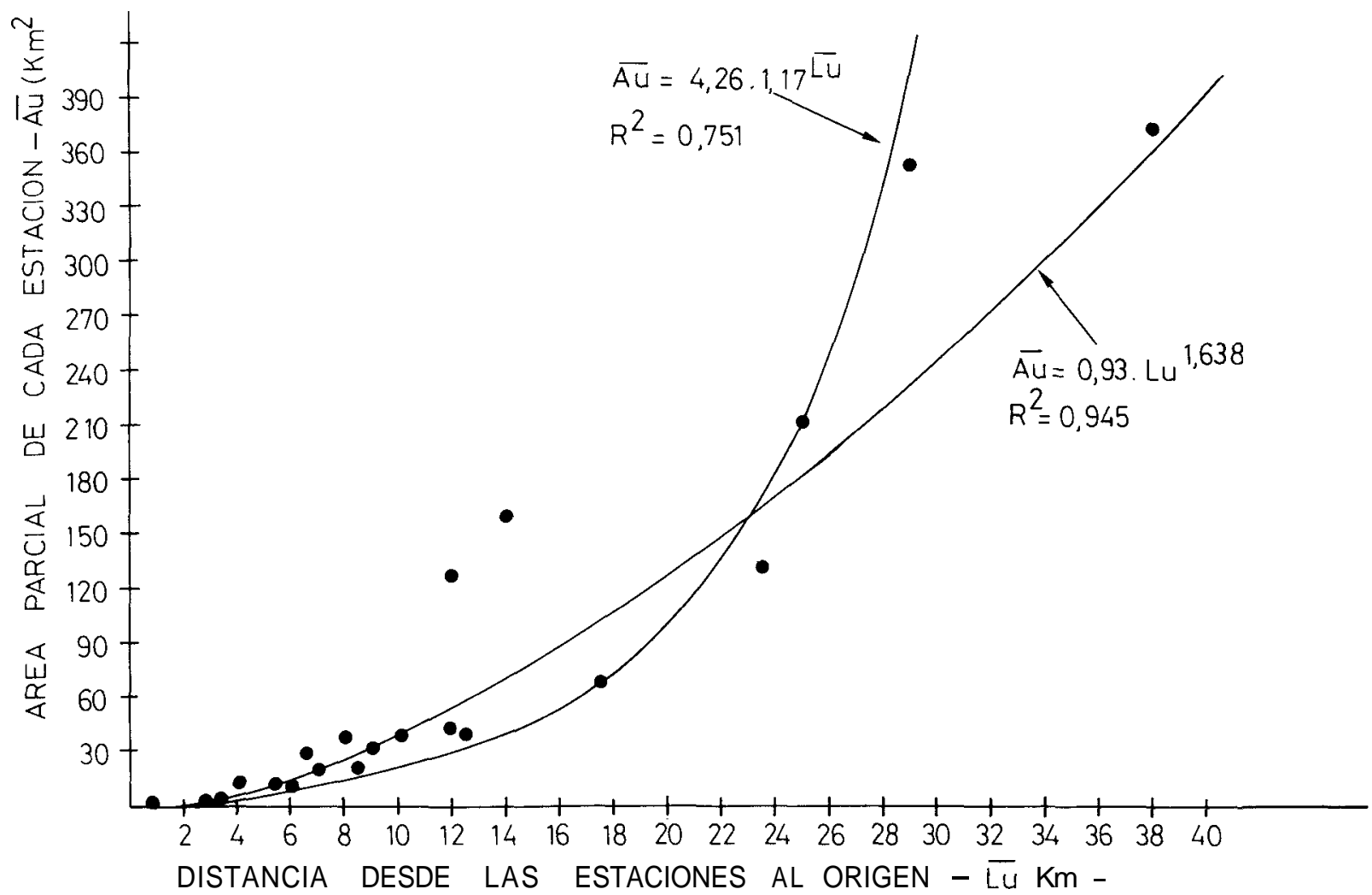

Figura 3.- Aplicación de las leyes de crecimiento alométrico y de crecimiento exponencial a la cuenca del río Ibaizabal. Ľu: longitud media de drenaje; Au: área parcial ocupada por los cauces de cada orden u; r: coeficiente de correlación de Pearson. Application of the laws of allometric growth and exponential growth to the Ibaizabal's basin. Lu: mean drainage length. Au: area occupied by segments of a determined degree; r: Pearson's correlation coefficient. 
cuencas: $b \simeq 1,6$ (Hynes, 1970; Prat et al. 1982); las cuencas del Cadagua, Butrón y Oka se encuentran en un estado de erosión intermedio; y, por último, un tercer grupo englobaría al resto de las cuencas, con valores de b inferiores a 1,40 . Dentro de este último grupo, el valor más bajo de alometría negativa lo presenta la cuenca del Asua, lo que indicaría que es una cuenca alargada y aproximadamente rectangular $(\mathrm{Ca}=1,74$, $\mathrm{b}=1,252)$, con un estado de erosión poco evolucionado.

Un caso particular lo constituye la cuenca del Artibai, donde el valor obtenido de la correlación entre $A u$ y $i \mathrm{u}$ ha sido el más bajo $(\mathrm{r}=0,655)$, aunque estadísticamente significativo $(\mathrm{p}<0,05)$, siendo también significativa la ecuación de regresión logarítmica $(\mathrm{t}=15,73 ; \mathrm{g} .1 .=8 ; \mathrm{p}<0,001)$. Sin embargo, el coeficiente de determinación $\mathrm{r}^{2}$ es muy bajo $\left(r^{2}=0,43\right)$, lo cual indica que el grado de ajuste del área como función potencial de la longitud de los segmentos de drenaje es débil. Por otro lado, b es $<1$ siendo el crecimiento muy alométrico negativo y, por lo tanto, $\mathrm{H}$ disminuye al aumentar iu. Hasta Urberuaga, en el eje principal y a una distancia de $11,5 \mathrm{~km}$ del origen (lo que representa el $62 \%$ de la longitud del río Artibai), la cuenca es de forma semicircular; a partir de dicha estación el área de drenaje se estrecha drásticamente, estableciendo frontera con el río Urio (afluente del Lea), lo que es debido a la existencia del monte Bardatzandi (701 m s.n.m.), y se ve corroborado por los valores de los índices de Gravelius y Caquot, ya comentados anteriormente.

En el estudio del crecimiento relativo de las cuencas consideramos tan importante conocer el tipo de crecimiento como cuantificarlo. Para ello proponemos la utilización de la curva de crecimiento exponencial (SNEDECOR \& Cochran, 1980), de uso muy frecuente en ecología. Esta ley dice que el ritmo de aumento, en cualquier etapa, es proporcional al tamaño ya alcanzado:

$$
\begin{gathered}
\frac{d \bar{A} u}{d \bar{L} u}=h \bar{A} u \\
\bar{A} u=c D^{\bar{L} u}=c e^{n t . u}
\end{gathered}
$$

Tal que $h=\ln D$, h representa el ritmo relativo constante de aumento, es decir, la tasa de crecimiento relativo $\left(\mathrm{n}^{\circ}\right.$ de $\mathrm{km}^{2}$ en que aumenta el
Tabla 6.- Determinación de la tasa relativa de crecimiento (h) de las principales cuencas fluviales de Bizkaia. Aplicación de

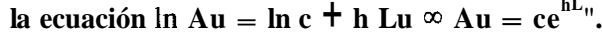

Estimation of the relative growth rate $(h)$ in Biscay's fluvial basins. Application of the equation $\ln \mathrm{Au}=\ln \mathrm{C}+$

\begin{tabular}{|c|c|c|c|c|c|}
\hline \multirow{3}{*}{$\frac{\text { Cuenca }}{\text { Carranza }}$} & $c$ & $h$ & $r$ & \multirow{3}{*}{$\frac{n}{5}$} & \multirow{3}{*}{$\frac{p}{<0,02}$} \\
\hline & \multicolumn{3}{|c|}{$\left(\mathrm{km}^{2} / \mathrm{km} / \mathrm{km}^{2}\right)$} & & \\
\hline & 2,29 & 0,27 & 0,951 & & \\
\hline Agüera & 9,58 & 0,14 & 0,999 & 3 & $<0,02$ \\
\hline Mercadillo & 2,43 & 0,19 & 0,921 & 9 & $<0,001$ \\
\hline Cadagua & 8,01 & 0,095 & 0,92 & 15 & $<0,001$ \\
\hline Galindo & 0,62 & 0,42 & 0,965 & 4 & $<0,05$ \\
\hline Nervión & 10,38 & 0,09 & 0,833 & 19 & $<0,001$ \\
\hline Ibaizabal & 4,26 & 0,15 & 0,866 & 33 & $<0,001$ \\
\hline Gobelas & 0,82 & 0,43 & 0,909 & 7 & $<0,005$ \\
\hline Asua & 0,7 & 0,4 & 0,914 & 8 & $<0,005$ \\
\hline Butrón & 0,54 & 0,19 & 0,903 & 20 & $<0,001$ \\
\hline Oka & 2,24 & 0,27 & 0,804 & 15 & $<0,001$ \\
\hline Lea & 1,6 & 0,19 & 0,813 & 11 & $<0,005$ \\
\hline Artibai & 5,47 & 0,1 & 0,64 & 10 & $<0,05$ \\
\hline
\end{tabular}
h Lu $\propto \mathrm{Au}=\mathrm{ce}^{\text {hLu }}$.

área de una cuenca por cada $\mathrm{km}$ de la red de drenaje y por cada $\mathrm{km}^{2}$ de área ya adquirida).

Las ecuaciones (8) y (12), además de ser matemáticamente distintas, expresan conceptos diferentes. Una aplicación de ambas se muestra en la fig. 3 para la cuenca del Ibaizabal. La ecuación (8) indica la «actividad cinética» del área de la red de drenaje, esto es, velocidad de crecimiento uniforme (isometría) o crecimiento acelerado (alometría), mientras que la función (12) determina en que cantidad constante se incrementa el área de la cuenca.

Entre los valores de b y h (tabla 6), la correlación no es significativa $(\mathrm{r}=0,22 ; \mathrm{g} .1 .=9$; $\mathrm{p}>0,1)$, lo cual implica que hay cuencas cuyo crecimiento es muy rápido pero pequeño y viceversa.

La velocidad relativa de crecimiento depende principalmente de la textura, protección del terreno y sobre todo de la topografía, mientras que el incremento del área depende de la intensidad de erosión, especialmente de la textura. En este sentido, las cuencas que tienen una tasa $\mathrm{h}$ más alta son las del Gobelas, Asua y Galindo, donde destacan materiales blandos como los aluviones, arcillas, arenas y margas. Los valores más bajos de $\mathrm{h}$ se han obtenido en las zonas constituidas por materiales más duros, fundamentalmente, basaltos, granitos, argilolitas, cuarzoarenitas etc. Tal 
es el caso del Agüera, Mercadillo, Cadagua, Nervión, Ibaizabal, Butrón, Lea y Artibai.

Hay que señalar que la rectificación logarítmica de los valores de Au frente a Lu se ajusta estadísticamente mejor a la curva potencial que a la exponencial, siendo la ecuación (8) mucho más exacta. Sin embargo, existe un porcentaje de variabilidad $\left(1-r^{2}\right)$ del área en función de la longitud que no se ajusta a las dos curvas. Esto puede deberse a condiciones muy concretas o puntuales de textura, topografía, microclimáticas e incluso a errores de muestreo estadístico. Este es el caso de la cuenca del Ibaizabal (fig. 3), en la que existen dos puntos que no se ajustan a ninguna de las dos curvas, dando lugar a un grado de indeterminación de $24,9 \%$ en la curva exponencial y del $5,5 \%$ en la potencial. Normalmente los puntos que más se ajustan a las dos curva son los correspondientes a los ríos de $1^{\circ}$ y $2^{\prime \prime}$ orden, que son precisamente a los que se debe principalmente el crecimiento del área de drenaje (fig. 3 ).

\section{Densidad de drenaje}

La densidad de drenaje (Dd) determina la longitud total de los cauces por unidad de superficie:

$\mathrm{D} d=\sum_{i=0}^{\mathrm{n}} \mathrm{L} \mathrm{u} / \mathrm{S}\left(\mathrm{km} / \mathrm{km}^{2}\right)$

Su valor está controlado por las características litológicas (especialmente la permeabilidad), la estructura de los materiales, tipo y densidad de vegetación y clima.

En nuestro estudio ninguna cuenca sobrepasa una densidad de $1 \mathrm{~km}$ por cada $\mathrm{km}^{2}$ de cuenca (tabla 7). En general toda Bizkaia tiene una red fluvial muy poco densa: aproximadamente 0,65 $\mathrm{km} / \mathrm{km}^{2}$ de superficie provincial. Este hecho puede deberse, entre otros, a dos factores principales:

1) La infiltración del agua bajo la superficie del terreno, fundamentalmente en los de naturaleza cárstica, generalmente formados por calizas arrecifales. En el área de estudio las cuencas más afectadas por este factor son: el tramo final de la cuenca del Carranza, que se ve influido por el complejo cárstico de Ramales, Calera, área del Duranguesado, cabecera del Nervión (este río nace en la zona cárstica de Sierra Salvada) y gran parte de las pequeñas cuencas costeras (Dd es muy bajo en las del Andrakas, Estepona, Artigas, Sollube, Laga y Ea). Por otro lado, también destacan las arcillas y margas, que proporcionan una gran escorrentía, lo cual implica poca consistencia del terreno y da lugar a valores bajos de densidad de drenaje.

2) La densa cobertura vegetal en las zonas rurales, formada principalmente por pinos, hayedos, encinares, robledales de distribución puntual, prados y vegetación azonal, fundamentalmente alisos ligados al curso de los ríos (OnAINDIA, 1986). En este sentido, García Rodríguez et al. (1984), en un estudio realizado en un área montañosa del centro-oeste de la península sobre paisaje y erosión, encuentra que la destrucción de la cubierta vegetal debida a un sobrepastoreo y deforestación intensos desestabiliza el ciclo hidrológico, incrementándose la densidad de drenaje y la erosividad potencial.

Tabla 7.- Cuantificación de la densidad de drenaje (Dd), constante de mantenimiento del canal (Mc) y frecuencia de los cauces $(\mathrm{Fu})$. Se dan también los valores de caudal medio hiperanual correspondientes a los anos 1970-1981.

Values of the drainage density (Dd), the channel's maintenance constant $(\mathrm{Mc})$ and frequency of the streams (Fu). Annual caudal mean values are also given, appertaining to the years 1970-1981.

\begin{tabular}{lcccc}
\hline Cuenca & $\begin{array}{c}D d \\
\left(\mathrm{~km} / \mathrm{km}^{2}\right)\end{array}$ & $\boldsymbol{M c}$ & $\boldsymbol{F u}$ & $\begin{array}{c}Q \\
\left(\mathrm{~m}^{3} / \mathrm{sg}\right)\end{array}$ \\
\hline Calera & $\mathbf{0 , 7 7}$ & 1,29 & 0.21 & 0,2 \\
Carranza & $\mathbf{0 , 6 2}$ & 1,61 & 0.21 & 1,5 \\
Agüera & 0,67 & 1,49 & 0,27 & 1,9 \\
Mercadillo & 0,56 & 1,78 & 0.18 & 0,2 \\
Cadagua & 0,57 & 1,75 & 0.23 & 1,82 \\
Galindo & 0,47 & 2.12 & 0,19 & - \\
Nervión & 0,61 & 1,63 & 0,22 & 25,31 \\
Ibaizabal & 0,64 & 1,56 & 0,38 & 8,5 \\
Asua & 0,91 & 1,09 & 0,36 & - \\
Gobelas & 0,82 & 1,21 & 0,24 & - \\
Butrón & 0,86 & 1,16 & 0,3 & 2,94 \\
Andrakas & 0.31 & 3,22 & 0,15 & - \\
Estepona & 0,36 & 2,77 & 0,14 & - \\
Artigas & 0,2 & 5 & 0,12 & - \\
Sollube & 0,42 & 2,38 & 0,12 & - \\
Oka & 0,99 & 1,01 & 0,33 & 1,29 \\
Laga & 0,43 & 2,32 & 0,03 & - \\
Ea & 0,31 & 3.22 & 0,15 & - \\
Lea & 0,76 & 1,31 & 0,36 & 0.72 \\
Artibai & 0,66 & 1,51 & 0,22 & 2,78 \\
Ego & 0,81 & 1,23 & 0,38 & - \\
\hline
\end{tabular}


En nuestra área de estudio, altas densidades de vegetación se dan en las cabeceras del Carranza, Mercadillo, Galindo, Butrón, Oka, Lea y Artibai.

En cierto grado, los valores bajos obtenidos de la tasa de crecimiento relativo (h) confirman los de la densidad de drenaje. Según la clasificación de STrahler (1977), la baja densidad de drenaje de la red fluvial de Bizkaia sería indicadora de una litología dominada por materiales de textura grosera. En este sentido, el perfil longitudinal de los ríos principales pone de manifiesto rupturas a distintas cotas, que pueden asignarse a una superposición de etapas erosivas o a la influencia litológica; sin embargo, la causa concreta de dichas rupturas en cada cuenca requiere de un análisis geológico que queda fuera de los objetivos de este trabajo. Lo que sí podemos afirmar es que las curvas de pendientes varían mucho de unas cuencas a otras, no existiendo correlación significativa entre los valores de pendiente y altitud de las estaciones de muestreo (Docampo \& Rallo, 1987b), lo cual pone de manifiesto la gran heterogeneidad geomorfornétrica de las distintas cuencas, sin que pueda hablarse de un patrón o unidad morfológica de los ríos de Bizkaia (en estudios que se están realizando actualmente sobre la fisicoquímica de las aguas, se han establecido 5 grupos, en cuanto a la mineralización del agua se refiere, muy relacionados con la composición litológica y la variabilidad geomorfológica).

La constante de mantenimiento del canal (Mc) es el inverso de la densidad de drenaje, de modo que representa el área necesaria para mantener una unidad de longitud de cauce. Según Sparks (1972), este parámetro puede ser utilizado para predecir si hay alguna posibilidad de que el sistema de drenaje continue extendiéndose hacia la cabecera, con el consiguiente peligro de deslizamientos. Los valores altos de Mc (tabla 7), son indicativos de cuencas con escasas posibilidades de extensión en las cabeceras, tal y como sucede en los pequeños ríos costeros (Artigas, Andrakas, Estepona, Sollube, Ea y Laga).

La frecuencia de cauces $(\mathrm{Fu})$ se define como el número de cauces $(\mathrm{Nu})$ por unidad de superficie:

$$
\mathrm{Fu}=\mathrm{Nu} / \mathrm{S}
$$

Este parámetro, al igual que Dd, es una medida de la textura del drenaje y, en consecuencia, del grado de disección del paisaje. En este sentido, los valores de Fu indicados en la tabla 7 corroboran los resultados obtenidos con la densidad de drenaje.

\section{DISCUSIÓN}

La utilización de los índices de Gravelius y de Caquot nos ha permitido determinar 12 cuencas o subcuencas cuya área está distribuida dentro de un perímetro regular: son las cuencas del Calera, Agüera, Mercadillo, Cadagua, Nervión, Ibaizabal, Gobelas, Butrón, Oka, Lea, las pequeñas cuencas costeras del Andrakas y Sollube, predominando las de forma alargada y circular.

En el estudio de la jerarquización de las redes fluviales se ha podido comprobar el cumplimiento de las leyes de Horton, esto es, una disminución creciente del número de segmentos al aumentar el grado, y una unidireccionalidad creciente; esto representa un ejemplo de la estrategia que gobierna la organización física del espacio en las cuencas.

VANNOTE et al (1980) establecen que, normalmente, entre los segmentos de $3^{\prime \prime}$ y $4^{\prime \prime}$ orden, un río pasa de ser un sistema heterotrófico a autotrófico. Anderson \& Sedell (1979), por otro lado, sitúan en el paso del 4" al 6" orden la zona en donde la proporción de material orgánico disuelto o en forma coloidal substituye al material orgánico grosero predominante en segmentos de río de orden inferior. En esta transformación intervienen la acción de la energía cinética (turbulencia y acción mecánica trituradora de las aguas), así como los propios organismos, tales como bacterias, hongos y detritívoros, que junto con la deriva directa o indirecta, contribuyen a la maduración biológica del río. Palau \& Palomes (1986) apuntan que cuando el río Segre pasa del 3' al 4" orden se hace más ancho y caudaloso, lo que permite un mayor desarrollo de macrófitos y un cambio en los grupos tróficos de macroinvertebrados bénticos.

En Bizkaia, la jerarquización de las redes fluviales es muy variable de unas cuencas a otras, y ello representa una gran heterogeneidad espacial o de hábitats. Así, tenemos que, en los ríos Agüera, Mercadillo, Galindo, Butrón y Oka, la vía au- 
tótrofa se da ya en los segmentos de $3^{\circ}$ orden. En los ríos Bolúe (Gobelas) y Golako (Oka), el mayor desarrollo de macrófitas y la mayor concentración de materia orgánica disuelta tiene lugar en segmentos de 2 " orden, lo cual coincide con un gradiente positivo de mineralización y eutrofización aguas abajo (GARCÍA DE BikUÑa et al, 1987). Por el contrario, en el río Lea, cuyo tramo final es de orden 4 , la vía heterotrófica se mantiene desde la cabecera hasta la entrada en la ría, siendo los aportes alóctonos muy abundantes a lo largo de toda su red, si bien, condiciones autotróficas se manifiestan estacionalmente en los remansos situadas en el tramo medio, aguas abajo de las numerosas represas de utilización agrícola existentes. En este río, la granulometría fina refleja el predominio de las gravas $(66,49 \% \pm 15,43 \% ; n=40)$; la vegetación de ribera está constituida por alisos, fresnos y avellanos, principalmente, con una cobertura arbórea entre el $80 \%$ y $100 \%$, siendo un río de mineralización media y niveles bajos de eutrofización; y, en cuanto a los organismos, se ha comprobado la ausencia de ciprínidos micrófagos, así como una alta diversidad trófica (GARCía DE BIKUÑA et al, op. ct.; Docampo \& RALlo, 1987a, 1987b).

En el estudio del crecimiento relativo, se observa que todas las cuencas experimentan, en mayor o menor grado, alometría negativa (en la mayoría alargamiento de la red de drenaje y estrechamiento del área drenada), con tendencia a un sistema de erosión maduro, que tendrá lugar cuando $b=2$. En este sentido, $b$ mide la velocidad relativa de crecimiento del área en función de la longitud de drenaje, poniendo de manifiesto deformaciones originadas durante el desarrollo, como en el caso del Artibai. Por otro lado, se propone la utilización de la curva de crecimiento exponencial con el fin de determinar la tasa de incremento relativo, la cual está relacionada principalmente con la naturaleza del substrato geológico.

Los valores de la densidad de drenaje y de la frecuencia de los cauces indican, por un lado, la textura grosera, y por otro, la gran protección que proporciona la cubierta vegetal en las zonas escasamente urbanizadas, sin hacer relación al tipo de caudales transportados por los ríos.

Por último, el estudio morfométrico de cuen- cas en base al cálculo de parámetros como densidad de drenaje, integral hipsométrica, constante de mantenimiento del canal etc. (SPARKS, op. cit.) aporta unos conocimientos de las mismas de gran utilidad para determinar riesgos de avenidas, deslizamiento ,etc., además de una información complementaria y necesaria, tanto en la planificación de estudios limnológicos como en el conocimiento del funcionamiento de los ríos en su aspecto biológico.

\section{AGRADECIMIENTOS}

Este trabajo ha podido ser realizado gracias a la subvención que la Exma. Diputación Foral de Bizkaia ha concedido al Proyecto de «Caracterización Fisico-Química y Biológica de la Red Hidrográfica de Bizkaia». En la determinación de los números de orden de las estaciones, así como en la obtención de las áreas de las cuencas participaron María Cacho y Ana Basaguren.

\section{Bibliografía}

Anderson, N.H. \& J.R. SEDELL, 1979. Detritus processing by macroinvertebrates in stream ecosystems. Ann. Rev. Entomol. 24: 351-377.

Antiguedad, I. \& J. Cruz-Sanjulián, 1980. Estudio morfométrico de la cuenca del río Arratia (Vizcaya). Boletín de la Real Sociedad Geográfica 66: 31-52.

Cruz-Sanjuliân, J. \& F. SÁEnz, 1980. Análisis cuantitativo de la red de drenaje de la cuenca alta del río Nervión. $K o$ bie (Ser. Ciencias Naturales) 10: 39-52.

Docampo, L., C. DEL CAMPo \& E. Rico, 1987. Investigaciones sobre el tritón jaspeado, Triturus marmoratus (Latreille, 1768), en el norte de España. Actas Congr. Biol. Ambiental (II Congreso Mundial Vasco), 2: 129-136:

Docampo, L. \& A. Rallo, 1987a. Tipología de las comunidades de vertebrados (peces y anfibios) de la red hidrográfica de Vizcaya. I Asociaciones entre especies y distribución de las cuencas. Kobie (Ser. Ciencias Naturales) 16: 257-267.

Docampo, L. \& A. Rallo, 1987b. Tipología de las comunidades de vertebrados (peces y anfibios) de la red hidrográfica de Vizcaya. II. Zonación de cuencas. Estructura trófica de las estaciones y correlaciones de las especies con la altitud, pendiente y temperatura. Kobie (Ser. Ciencias $\mathrm{Na}$ turales) 16: 269-290 
Eraso, A. \& I. Antiguedad, 1984. Estudio de la transformación «precipitación-aportación* en relación con los parámetros físicos de las cuencas hidrográficas. Aplicación a cuatro cuencas de Bizkaia. Lurralde (Investigación y Espacio) 6: 173-195.

García Rodriguez, J.A., A. Puerto Martín \& R. RodríGUEZ GONZÁLEZ, 1984. Aplicación de un método sencillo de análisis hidrográfico al estudio ecológico del paisaje y la erosión en un área montañosa del centro-oeste ibérico. Studia Oecológica 5: 301-322.

García de Bikuña, B., A. Basaguren, M. Cacho \& E. ORIVE, 1987. Características fisico-químicas de las aguas superficiales de los principales ríos de Bizkaia. Actas del $\mathrm{N}$ Congreso Español de Limnología: 165-177.

HorTON, R.E., 1945. Erosional development of streams and their drainage basins: hydrophysical approach to quantitative morphology. Bull. Geol. Soc. Am. 56: 275-370.

HYNES, H.B.N., 1970. The Ecology of running waters. University Press. Liverpool. $555 \mathrm{pp}$.

JiMÉNEZ, J. \& J . MARTíneZ-LÓPEZ, 1988. Distribución y composición específica de la malacofauna del río Júcar. Limnetica 4: 9-18.

Margalef, R., 1974. Ecología. Omega. Barcelona. 951 pp.

ONAINDfA, M., 1986. Ecología vegetal de las Encartaciones y Macizo del Gorbea. Vizcaya. 271 pp. Bilbao. Universidad del País Vasco.

Palau A., 1987. Caracterización morfométrica de la cuenca catalana del río Segre (Lérida, NE España). Actas del IV Congreso Español de Limnología: 147-154.

Apéndice.- Descripción de las estaciones de muestreo Description of sampling sites.
Palau, A. \& A. Palomes, 1986. Diagnóstico fisico-químico y biológico del Río Segre. Dptos. Ciencia del Suelo, Climatología y Microbiología. E.T.S.I.A. Lleida. 500 pp.

Prat, N., M.A. Puig, G. González \& M.J. Tort, 1982. Predicció $i$ control de la qualitat de les aigües dels rius Besós $i$ Llobregat. I. Els factors físics i químics del medi. Servei del Medi Ambient. Diputació de Barcelona. Barcelona. 206 pp.

RAYNAL, R., 1970. Geomorphologie et vocation des sols dans le pays du bassin occidental de la Méditerranée. Ann. Univ. Bucaresti Geogr. 19: 21-33.

Remenieras, G., 1971. Tratado de hidrología aplicada. Técnicos Asociados. Barcelona. $515 \mathrm{pp}$.

Roche, M., 1963. Hydrologie de surface. Gauthier-Villars. París. 430 pp.

SABATER, F., 1987. Estudi integral del riu Ter i la seva conca: Les característiques de l'aigua i els factors que les determinen. Tesis Doctoral. Universitat de Barcelona.

Schumm S.A., 1956. The evolution of drainage systems and slopes in bad-lands at Perth Amboy, New Jersey. Bull. Geol. Soc. Am. 67: 597-646.

SnedeCor, G.W. \& W.G. Cochran, 1980. Métodos estadísticos. Compañía Editorial Continental. México. 703 pp.

Sparks, B.W.,1972. Geomorphology. Longman Group. Ltd. London. $530 \mathrm{pp}$.

STRAHLER, A.N., 1952. Hypsometric analysis of erosional topography. Bull. Geol. Soc. Am. 63: 923-938

Strahler, A.N., 1977. Geografía física. Ed. Omega. Barcelona. $767 \mathrm{pp}$.

Vannote, R.L., G.W. Minshall, K.W. Cummins, J.R. SeDELL \& C.E. CusHing, 1980. The river continuum concept. Can. J. Fish. Aquatic. Sci. 37: 130-137.

\begin{tabular}{|c|c|c|c|c|c|c|c|c|}
\hline Cuenca & Río & Localidad & Código & U.T.M. & $D$. & A. & P.\% & Geología \\
\hline \multirow[t]{6}{*}{ ASON } & Calera & Lanestosa & CA1-1 & 30TVB650835 & 10 & 315 & 4 & Calizas arcillosas, areniscas \\
\hline & Argañeda & Lanzas Agudas & CAr-1 & $30 T V N 708818$ & 5 & 260 & 2 & Arcillas y areniscas \\
\hline & Escaleras & Pando & CE-1 & 30TVN741831 & 4 & 300 & 8 & Arcillas y areniscas \\
\hline & Escaleras & Concha & CE-2 & $30 \mathrm{TVN} 713855$ & 9 & 180 & 4 & Aluviones \\
\hline & Callojuolas & Horboso & $\mathrm{CCa}-1$ & 30 TVN738892 & 1 & 230 & 6 & Arcillas hojosas \\
\hline & Carranza & Carranza & $\mathrm{C}-1$ & $30 T V N 699885$ & 13 & 140 & 2 & Calizas arcillosas, areniscas \\
\hline \multirow[t]{3}{*}{ AGUERA } & Agüera & El Solar & $A G=1$ & $30 \mathrm{TWN} 782867$ & 3,5 & 220 & 4 & Aluviones, arcillas arenosas y arcillas \\
\hline & Agüera & La Iglesia & $\mathrm{AG}-2$ & 30TVN793908 & 8,5 & 140 & 1,3 & $\begin{array}{l}\text { Aluviones, margas hojosas. calizas ar- } \\
\text { cillosas con areniscas }\end{array}$ \\
\hline & Agüera & Agüera & $A G-3$ & $30 T V N 792937$ & 12 & 120 & 0,8 & $\begin{array}{l}\text { Areniscas y calizas con orbitolinas. } \\
\text { margas arenosas }\end{array}$ \\
\hline
\end{tabular}




\begin{tabular}{|c|c|c|c|c|c|c|c|c|}
\hline Cuenca & Río & Localidad & Código & U.T.M. & $D$. & A. & $P . \%$ & Geología \\
\hline \multirow[t]{9}{*}{ MERCADILLO } & Gotitza & Arcentales & MG-1 & 30 TVN 823873 & 4,5 & 220 & & $\begin{array}{l}\text { Areniscas, margas y areniscas calcá- } \\
\text { reas }\end{array}$ \\
\hline & Avellaneda & Avellaneda & MA-1 & 30TVN873871 & 1 & 220 & 4 & Margas y calizas de espículas \\
\hline & Galdames & San Pedro & MGa-1 & 30TVN919891 & 3,5 & 140 & 1,3 & Margas, calizas y areniscas \\
\hline & Golitza & Mercadillo & $\mathrm{MG}-2$ & 30 TVN871902 & 11 & 100 & 2 & Calizas, areniscas \\
\hline & Galdames & La Aceña & $\mathrm{MGa}-2$ & 30TVN903912 & 75 & 70 & 2,5 & Margas y areniscas \\
\hline & Tremoral & El Castaño & MT-1 & 30TVN868919 & 1 & 120 & 1,6 & Margas y aluviones \\
\hline & Mercadillo & Somorrostro & M-1 & 30TVN899942 & 18 & 20 & 1 & $\begin{array}{l}\text { Aluvión, arenisca, margas y areniscas } \\
\text { calcareas }\end{array}$ \\
\hline & Mercadillo & San Juan de S. & $\mathrm{M}-2$ & 30TVN903957 & 20 & 10 & & $\begin{array}{l}\text { Aluvión, arenisca, margas y areniscas } \\
\text { calcáreas }\end{array}$ \\
\hline & Cotorrio & Cotorio & MC-1 & 30 TVN922957 & 3,5 & 60 & & $\begin{array}{l}\text { Areniscas, margas y areniscas calcà- } \\
\text { reas }\end{array}$ \\
\hline \multirow[t]{15}{*}{ CADAGUA } & Cadagua & Cadagua & $\mathrm{Ca}-1$ & 30TVN719698 & 2,5 & 380 & 2 & Arcillas, yesos y cal \\
\hline & Ordunte & LaLosa & $\mathrm{CAO}-1$ & 30TVN719749 & 6 & 380 & 2 & Aluviones. margas y calizas arcillosas \\
\hline & Cadagua & Entrambasaguas & $\mathrm{CA}-2$ & $30 \mathrm{TVN} 784738$ & 12 & 280 & 1 & Aluviones, margas y calizas arcillosas \\
\hline & Ordunte & Vega de Nava & $\mathrm{CAO}-2$ & 30 TVN794797 & 16 & 220 & 1 & $\begin{array}{l}\text { Aluviones, areniscas y arcilla con orbi- } \\
\text { tolinas }\end{array}$ \\
\hline & Cadagua & Valmaseda & $\mathrm{Ca}-3$ & $30 \mathrm{TVN} 834812$ & 24 & 160 & 1,3 & $\begin{array}{l}\text { Aluviones, areniscas, arcillas arenosas } \\
\text { y arcillas con orbitolinas }\end{array}$ \\
\hline & Cadagua & Valmaseda & CA-4 & 30 TVN857836 & 27 & 120 & 1 & Areniscas \\
\hline & Cadagua & El Corrillo & CA-5 & 30 TVN892845 & 32 & 100 & 1 & Aluvión, margas y areniscas \\
\hline & Retola & Retola & CAR-1 & $30 \mathrm{TVN} 859857$ & 1 & 160 & 6 & Aluvión, margas y areniscas \\
\hline & Cadagua & Gücñes & CA-6 & 30TVN926842 & 36 & 80 & 1 & Aluvión, margas y calizas de espículas \\
\hline & Herrerias & Martijana & $\mathrm{CAH}-1$ & 30 TVN879677 & 15 & 340 & 2 & Margas y calizas arcillosas \\
\hline & Herrerias & Arza & $\mathrm{CAH}-4$ & 30 TVN863777 & 7,5 & 200 & 1 & Aluvión, margas y calizas \\
\hline & Herrerias & Albichu & $\mathrm{CAH}-2$ & 30TVN915775 & 28 & 120 & 1 & Aluvión, areniscas y arcillas \\
\hline & Herrerias & Sodupe & $\mathrm{CAH}-3$ & 30TVN963825 & 36 & 80 & 1 & $\begin{array}{l}\text { Aluvión, margas y calizas con espícu- } \\
\text { las }\end{array}$ \\
\hline & Cadagua & La Cuadra & CA-7 & 30TVN982858 & 46 & 60 & 1 & Aluvión, margas y areniscas \\
\hline & Cadagua & Alonsótegui & CA-8 & $30 \mathrm{TWN} 023893$ & 53 & 20 & 1 & $\begin{array}{l}\text { Aluviones, areniscas, margas y arenis- } \\
\text { cas calcáreas }\end{array}$ \\
\hline \multirow[t]{4}{*}{ GALINDO } & Galindo & El Regalo & GA-1 & 30TVN984897 & 5 & 80 & 10 & $\begin{array}{l}\text { Areniscas, margas y areniscas calcá- } \\
\text { reas }\end{array}$ \\
\hline & Galindo & Retuerto & GA-2 & 30 TWN007921 & 9,5 & 20 & 1 & Aluvión, margas y areniscas \\
\hline & Capetillo & Nocedal & GAC-1 & 30TVN967955 & 1 & 40 & 2 & Margas y calizas de cspículas \\
\hline & Vallontí & E1 Mello & GAV-1 & 30TVN966967 & 2 & 40 & 2 & Margas y calizas de espículas \\
\hline NERVIÓN / & Nervión & Délica & $\mathrm{N}-1$ & 30 TWN016540 & 3 & 800 & 1,5 & Margas, calizas arcillosas y calizas \\
\hline \multirow[t]{15}{*}{ IBAIZABAL } & Tertanga & Tertanga & NT-1 & 30 TVN982584 & 2,8 & 340 & 4 & $\begin{array}{l}\text { Calizas y margas, arcillas irisadas, ye- } \\
\text { sos y sal gema }\end{array}$ \\
\hline & Nervión & Délica & $\mathrm{N}-2$ & $30 \mathrm{TWN} 007579$ & 8 & 300 & 1,3 & Arcillas irisadas, yesos y sal gema \\
\hline & Nervión & Orduña & $\mathrm{N}-3$ & 30TVN996613 & 12 & 280 & 1 & Arcillas abigarradas y yesos \\
\hline & Nervión & Luyando & $\mathrm{N}-4$ & 30TVN999713 & 22 & 180 & 1 & Aluviones y areniscas \\
\hline & Altube & & NA-1 & $30 \mathrm{TWN} 078623$ & 7,5 & 340 & 1.66 & $\begin{array}{l}\text { Areniscas, arcilla, caliza y conglome- } \\
\text { rados }\end{array}$ \\
\hline & Altube & Altube & NA-2 & 30TWN064695 & 17 & 320 & 1.2 & $\begin{array}{l}\text { Areniscas, arcilla, caliza y conglome- } \\
\text { rados }\end{array}$ \\
\hline & Ibarra & Ibarra & NI-1 & $30 T W N 121712$ & 5 & 260 & 1,2 & Calizas \\
\hline & Arnauri & Ibarra & NAr-1 & 30TWN114715 & 5 & 260 & 1,2 & Calizas \\
\hline & Arnauri & Orozco & NAr-2 & 30TWN077727 & 9 & 180 & 1 & Calizas \\
\hline & Altube & Orozco & NA-3 & $30 T W N 074738$ & 22 & 160 & 1 & Calizas \\
\hline & Altube & Areta & $\mathrm{NA}-4$ & $30 T W N 051764$ & 26 & 120 & 1 & Aluviones, arcillas, areniscas y margas \\
\hline & Ceberto & Ceberto & $\mathrm{NC}-1$ & $30 \mathrm{TWN} 146774$ & 2 & 180 & 2 & Argilitas \\
\hline & Gorozitu & Ceberto & NG-1 & 30TWN139781 & 2,3 & 180 & 2 & Argilitas \\
\hline & Ceberio & Miravalles & $\mathrm{NC}-2$ & 30TWN088796 & 9 & 80 & 1,6 & Argilitas, calizas y margas \\
\hline & Nervión & Barakaldo & $\mathrm{N}-5$ & 30TWN050781 & 32 & 120 & 1 & Aluvión, arcillas y areniscas \\
\hline
\end{tabular}




\begin{tabular}{|c|c|c|c|c|c|c|c|c|}
\hline Cuenca & Río & Localidad & Código & U.T.M. & $D$. & $A$. & P. \% & Geología \\
\hline & Larumbe & Zoilo & NL-1 & 30TWN045818 & 1,5 & 160 & 3,3 & Margas y areniscas \\
\hline & Nervión & Miravalles & N-6 & $30 \mathrm{TWN} 084824$ & 43 & 40 & 1 & $\begin{array}{l}\text { Aluvión, areniscas, margas y areniscas } \\
\text { calcáreas }\end{array}$ \\
\hline & Nervión & Basauri & $\mathrm{N}-7$ & 30TWN101877 & 51 & 10 & 1 & Aluvión, margas y areniscas \\
\hline & Ibaizabal & Zaldivar & $\mathrm{I}-1$ & $30 \mathrm{TWN} 381802$ & 0,8 & 260 & 6 & $\begin{array}{l}\text { Calizas margosas, arenas, areniscas y } \\
\text { calizas }\end{array}$ \\
\hline & Elorrio & Elorrio & IE-1 & 30 TWN382748 & 4 & 200 & 1,5 & Calizas \\
\hline & Elorrio & Elorrio & $\mathrm{IE}-2$ & $30 \mathrm{TWN} 364757$ & 6,5 & 180 & 1 & Calizas \\
\hline & Arrazola & Santiago & IAr-1 & $30 \mathrm{TWN} 339732$ & 5,5 & 200 & 1,3 & $\begin{array}{l}\text { Basaltos, areniscas, arcilla, caliza y } \\
\text { conglomerados }\end{array}$ \\
\hline & Atxarte & Atxarte & IAt-1 & $30 \mathrm{TWN} 297745$ & 3,5 & 300 & 2,8 & Calizas \\
\hline & Mañaria & Manaria & IMa-1 & $30 \mathrm{TWN} 274760$ & 4 & 220 & 2,8 & Argilitas \\
\hline & Elorrio & Abadiano & IE-3 & 30 TWN316784 & 14 & 140 & 1 & Calizas \\
\hline & Oka & Oka & IO-1 & $30 \mathrm{TWN} 350824$ & 2,5 & 300 & 8 & Calizas y arenas \\
\hline & San Cristóbal & Iturbe & IS-1 & $30 \mathrm{TWN} 324822$ & 4 & 200 & 5 & Tramo calcáreo \\
\hline & Ibaizabal & Durango & $\mathrm{I}-2$ & $30 \mathrm{TWN} 316802$ & 8 & 140 & 1 & Arenas, arcillas y limos \\
\hline & Ibaizabal & Mallabia & $\mathrm{I}-3$ & $30 \mathrm{TWN} 287809$ & 12 & 100 & 1 & \\
\hline & Magunas & Magunas & IM-1 & $30 \mathrm{TWN} 277851$ & 5 & 200 & 2 & Calizas arenosas y margas \\
\hline & Ibaizabal & Bernabeitia & $\mathrm{I}-4$ & $30 T W N 264817$ & 14 & 100 & 1 & $\begin{array}{l}\text { Arenas, limos, tramo calcáreo, argilo- } \\
\text { litas y cuarzoarenitas }\end{array}$ \\
\hline & Malaespera & Amorebieta & IMI-1 & $30 \mathrm{TWN} 229828$ & 1 & 120 & 9 & Areniscas, arcillas \\
\hline & San Martín & Amorebieta & ISm-1 & $30 \mathrm{TWN} 228857$ & 3,5 & 100 & 1,8 & Tramo calcáreo \\
\hline & Ibaizabal & Amorebieta & $\mathrm{I}-5$ & 30TWN201846 & 25 & 80 & 1 & $\begin{array}{l}\text { Arcillas y limos con argilolitas y cuar- } \\
\text { zoarenitas }\end{array}$ \\
\hline & Arratia & Barazar & IA-1 & $30 T W N 208692$ & 5 & 240 & 1,8 & Areniscas, arcillas y calizas \\
\hline & Arratia & Undurraga & IA-2 & $30 T W N 214701$ & 7 & 220 & 2 & Areniscas, arcillas y calizas \\
\hline & Arratia & Lezaga & IA-3 & 30 TWN204713 & 9 & 180 & 1,7 & Areniscas, arcillas y calizas \\
\hline & Indusi & Dima & IAI-1 & $30 \mathrm{TWN} 209761$ & 8,5 & 140 & 1,6 & Areniscas, arcillas y calizas \\
\hline & Indusi & Dima & IAI-2 & 30TWN194774 & 10 & 110 & 1 & Areniscas, arcillas y calizas \\
\hline & Gorbea & Villaro & IAG-1 & $30 T W N 173731$ & 2 & 300 & 13,4 & Areniscas, arcillas y calizas \\
\hline & Arratia & Villaro & IA-4 & 30TWN191735 & 12 & 180 & 1 & $\begin{array}{l}\text { Areniscas, arcillas, calizas y caliza re- } \\
\text { cital }\end{array}$ \\
\hline & Oba & Dima & IAO-1 & 30TWN207769 & 4 & 120 & 1 & Caliza recital \\
\hline & Arratia & Aranzazu & IA-5 & 30TWN171779 & 18 & 100 & 1 & Caliza recital \\
\hline & Indusi & Yurre & IAI-3 & 30TWN185786 & 13 & 100 & 1 & Argilolitas \\
\hline & Arratia & Larrabiti & IA-6 & $30 T W N 187833$ & 24 & 80 & 4 & Areniscas y arcillas \\
\hline & Larrea & Garaitondo & IL-1 & 30TWN205888 & 3 & 120 & 1,8 & Calizas arenosas y margas \\
\hline & Erkinkos & Larrabezua & IEr-1 & 30TWN189905 & 1 & 100 & 4 & Calizas arenosas y margas \\
\hline & Larrabezua & Larrabezua & ILa-1 & $30 \mathrm{TWN} 162889$ & 6 & 60 & 1 & Argilolitas y cuarzoarenitas \\
\hline & Borrao & Erleches & IB-1 & 30TWN165871 & 6,5 & 60 & 1 & Argilolitas y cuarzoarenitas \\
\hline & Ibaizabal & Lemona & $1-6$ & 30TWN173845 & 29 & 60 & 1 & $\begin{array}{l}\text { Arenas, arcillas, limos, caliza recifal y } \\
\text { argilolitas calcáreas }\end{array}$ \\
\hline & Ibaizabal & Olabarrieta & I-7 & 30TWN116866 & 38 & 40 & 1 & Aluvión, margas y areniscas calcáreas \\
\hline \multirow[t]{7}{*}{ ASUA } & Asua & Lezama & AS-1 & 30TWN145911 & 0,5 & 60 & 1,8 & Tramo calcáreo \\
\hline & Basobal & Lezama & ASB-1 & 30TWN143924 & 2,5 & 80 & 1,3 & Tramo calcáreo \\
\hline & Untza & Bidarte-Barri & ASU-1 & 30TWN099956 & 1 & 80 & 2,2 & Margas, calizas \\
\hline & Preguntegui & Sondica & ASP-1 & 30TWN087949 & 2,5 & 40 & 2 & Margas, calizas \\
\hline & Kantarasarra & Zamudio & ASK-1 & 30TWN11914 & 500 & 100 & 6,6 & \\
\hline & Asua & La Ola & AS-3 & 30TWN072932 & 11 & 20 & 1 & Aluviones y margas \\
\hline & Antontzune & Zabaloeche & ASA-1 & 30TWN052958 & 1,5 & 20 & 1 & Margas y calizas \\
\hline UDONDO & Udondo & Mendoche & UD-1 & 30TWN031966 & 1 & 40 & 1 & Aluviones y margas \\
\hline \multirow[t]{4}{*}{ GOBELAS } & Gobelas & Sopelana & $\mathrm{G}-1$ & 30TWP013038 & 1 & 40 & 2 & Aluvión, calizas arenosas y margas \\
\hline & Muñarrekolai & Larrondo & GM-1 & 30TWP023032 & 1,5 & 60 & 3,5 & Tramo calcáreo \\
\hline & Gobelas & Larrabasterra & G-2 & 30TWP003026 & 3 & 40 & 1 & Aluviones y crestones calcáreos \\
\hline & Eguskiza & Arrikapela & GE-1 & 30TWP014005 & 1 & 20 & 1 & Calizas y arenas \\
\hline
\end{tabular}




\begin{tabular}{|c|c|c|c|c|c|c|c|c|}
\hline Cuenca & Río & Localidad & Código & U.T.M. & $D$. & $A$. & $P . \%$ & Geología \\
\hline & Bolue & Lejona & GB-1 & 30TWN038989 & 2 & 40 & 2,2 & $\begin{array}{l}\text { Microconglomerados, areniscas, mar- } \\
\text { gas rojas y calizas }\end{array}$ \\
\hline & Bolue & Getxo & GB-2 & 30TWN018994 & 6 & 20 & 1 & Aluviones, calizas y arenas \\
\hline & Gobelas & Algorta & G-3 & 30TWN001998 & 7 & 20 & 1 & Aluviones, calizas y arenas \\
\hline \multirow[t]{20}{*}{ BUTRÓN } & Butrón & Oñarte & B-1 & 30TWN193936 & 0,5 & 200 & 7 & Tramo calcáreo \\
\hline & Butrón & Becobaso & B-2 & 30TWN201971 & 5,5 & 80 & 1 & Arcillas, arenas, limo y tramo calcáreo \\
\hline & Rigoitia & Rigoitia & BR-1 & $30 \mathrm{TWN} 226964$ & 0,5 & 240 & 2 & Zona calcárea \\
\hline & Rigoitia & Urrebarri & BR-2 & 30 TWN205981 & 3,5 & 100 & 1,5 & Basaltos y tramo calcáreo \\
\hline & Atxispe & Echevarri & BAt-1 & 30TWN169938 & 3 & 80 & 1 & Calizas arenosas y margas \\
\hline & Butrón & Fruniz & B-3 & $30 \mathrm{TWN} 173974$ & 9,5 & 60 & 1 & Arcillas, limo y tramo calcáreo \\
\hline & Bolintxu & Gamiz & BB-1 & 30TWN148968 & 2 & 40 & 1,2 & Arcillas, arenas, limo y tramo calcáreo \\
\hline & Sollube & Meñacabarrena & BS-1 & 30TWP170003 & 3 & 100 & 2 & Argilolitas y cuarzoarcnitas \\
\hline & Talleri & Larrakoetxe & BT-1 & 30TWP161024 & 2,5 & 80 & 1,7 & Tramo calcáreo \\
\hline & Larrauri & Larrauri & BL-1 & 30TWP143033 & 2 & 80 & 3,3 & Tramo calcáreo \\
\hline & Larrauri & Belako & BL-2 & 30TWN150993 & 7 & 30 & 1 & Aluvión, basaltos y tramo calcáreo \\
\hline & Butrón & Echevarri & B-4 & 30TWN134999 & 16 & 30 & & Aluvial y tramo calcáreo \\
\hline & Butrón & San Andrés & B-5 & 30TWP108019 & 22 & 20 & 1 & Aluvión y tramo calcáreo \\
\hline & Azioerreka & Azolo & BA-1 & 30TWP097046 & 1,5 & 60 & 2 & Argilolitas y cuarzoarenitas \\
\hline & Andrakas & San Lorenzo & Ban-1 & 30TWP086046 & 0,5 & 100 & 4 & Argilolitas y cuarzoarenitas \\
\hline & Butrón & Gatika & B-6 & 30TWP094023 & 25 & 20 & 1 & $\begin{array}{l}\text { Aluviones y tramo calcáreo (izda.), ar- } \\
\text { gilolitas y cuarzoarenitas (dcha) }\end{array}$ \\
\hline & Amazkari & Golzai & BAm-1 & 30TWP065006 & 1,5 & 20 & 1 & Zona calcárea \\
\hline & Errazkondo & Libarona & BE-1 & 30TWP088017 & 4 & 20 & 1 & Terreno calcáreo \\
\hline & Butrón & Butrón & B-7 & 30TWP072023 & 30 & 10 & 1 & Calizas arenosas y margas \\
\hline & Kukutxa & Learre & BK-I & 30TWP046032 & 2 & 60 & 1,9 & Zona calcárea \\
\hline ANDRAKAS & Andrakas & Arminza & AN-1 & 30TWP078081 & 3 & 50 & 2 & Argilolitas y cuarzoarenitas \\
\hline \multirow[t]{3}{*}{ ESTEPONA } & B. Infierno & Uribarri & ESI-1 & 30TWP184034 & 1 & 310 & 7,5 & Argilolitas y cuarzoarenitas \\
\hline & Carracola & Baquio & ESc-1 & 30TWP139063 & 1 & 160 & 7,5 & Calizas y argilolitas \\
\hline & Estepona & Baquio & ES-1 & 30TWP151065 & 7 & 20 & 4 & Argilolitas y cuarzoarenitas \\
\hline ARTIGAS & Artigas & Bermeo & AR-1 & 30TWP218054 & 1 & 60 & 2,1 & Argilolitas y cuarzoarenitas \\
\hline \multirow[t]{2}{*}{ BUSTURIA } & Sollube & Busturia & $\mathrm{SO}-1$ & 30TWP231028 & 3 & 60 & 3 & Argilolitas y cuarzoarenitas \\
\hline & Sollube & Busturia & $\mathrm{SO}-2$ & 30TWP238018 & 4,5 & 20 & 1 & Argilolitas y cuarzoarenitas \\
\hline \multirow[t]{15}{*}{ OKA } & Oka & Zugaztieta & O-1 & 30TWN253898 & 4,5 & 80 & 8 & Calizas arenosas y margas \\
\hline & Ibarruri & Ibarruri & OI-1 & $30 \mathrm{TWN} 265878$ & 2 & 140 & 4 & Calizas arenosas y margas \\
\hline & Oka & Mugica & $0-2$ & $30 \mathrm{TWN} 256916$ & 6,5 & 20 & 1 & Tramo detrítico \\
\hline & Oka & Campanchu & $0-3$ & 30 TWN262948 & 10 & 10 & 1 & Arenas, arcillas, limo y tramo calcárco \\
\hline & Txareta & Ugarte & OT-1 & 30 TWN233928 & 1,8 & 80 & 5 & Tramo detrítico \\
\hline & Eder & Guernica & OE-1 & $30 \mathrm{TWN} 244953$ & 1,5 & 30 & 3,3 & Tramo calcáreo \\
\hline & Baldatika & Guernica & OBa-1 & $30 \mathrm{TWN} 244974$ & 1,5 & 110 & 3,3 & Tramo calcáreo y basaltos \\
\hline & Berrakondo & Lamikiz & OB-1 & 30 TWN275913 & 2 & 60 & 2 & Tramo detrítico \\
\hline & Berrakondo & Guernica & OB-2 & 30TWN270941 & 5,5 & 10 & 1 & Arenas, arcillas, limos, yesos y ofitas \\
\hline & Golako & Inchaurreta & OG-1 & 30TWN29892 & 1 & 200 & 4 & Tramo dctrítico y tramo calcáreo \\
\hline & Golako & Arrazua & OG-2 & $30 \mathrm{TWN} 287957$ & 9,5 & 20 & 1 & $\begin{array}{l}\text { Arenas, arcillas, basaltos y tramos cal- } \\
\text { cáreos }\end{array}$ \\
\hline & Gaztiburu & Elorrieta & OGa-1 & $30 T W N 302938$ & 3 & 100 & 2,8 & \\
\hline & Golako & Guernica & OG-3 & $30 \mathrm{TWN} 275968$ & 12 & 10 & 1 & Arenas, arcillas, limos, yesos y ofitas \\
\hline & Huarka & Zabala-Belendiz & $\mathrm{OH}-1$ & $30 \mathrm{TWN} 294970$ & 3,5 & 80 & 3 & Argilolitas y cuarzoarenitas \\
\hline & Oma & Arteaga & $00-1$ & 30TWN286997 & 2 & 10 & 1 & Arcillas y yesos \\
\hline \multirow[t]{2}{*}{ LAGA } & Laga & Ibarrangelua & LAg-1 & $30 \mathrm{TWP} 295027$ & 1 & 140 & 4 & Argilolitas y cuarzoarenitas \\
\hline & Laga & Ibarrangelua & LAg-2 & 30TWP288054 & 5 & 40 & 2 & $\begin{array}{l}\text { Argilolitas y cuarzoarenitas (dcha), ca- } \\
\text { lizas y margas (izda) }\end{array}$ \\
\hline \multirow[t]{2}{*}{ EA } & Goikoetxe & $\mathrm{Ea}$ & EG-1 & 30TWP330030 & 2 & 20 & 1 & Argilolitas y cuarzoarenitas \\
\hline & $\mathrm{Ea}$ & $\mathbf{E a}$ & E-1 & 30TWP338027 & 4 & 20 & 1 & \\
\hline
\end{tabular}




\begin{tabular}{|c|c|c|c|c|c|c|c|c|}
\hline Cuenca & Río & Localidad & Código & U.T.M. & $D$. & $A$. & P.\% & Geología \\
\hline \multirow{11}{*}{ LEA } & Lea & Guerricaiz & L-1 & 30TWN337898 & 4 & 210 & 2 & Sustrato calcáreo, tramo detntico \\
\hline & Lea & Aulestia & $\mathrm{L}-2$ & 30TWN337931 & 9 & 100 & 2 & $\begin{array}{l}\text { Arenas, limos y arcillas, argilolitas y } \\
\text { cuarzoarenitas }\end{array}$ \\
\hline & Otz & Aulestia & LO-1 & 30 TWN352932 & 1 & 120 & & $\begin{array}{l}\text { Arenas, arcillas, limos, argilolitas cal- } \\
\text { cáreas }\end{array}$ \\
\hline & Lea & Aulestia & $\mathrm{L}-3$ & 30TWN372951 & 13 & 60 & & $\begin{array}{l}\text { Arenas, arcillas y limos, arcillas mar- } \\
\text { gosas }\end{array}$ \\
\hline & Asua & Aulestia & LA-1 & 30 TWN344940 & 1,5 & 100 & 1 & Argilolitas y cuarzoarenitas \\
\hline & Okanika & Guizaburuaga & LOK-1 & $30 \mathrm{TWN} 368981$ & 2 & 80 & 7 & Caliza paraarrecifal. \\
\hline & Lea & Guizaburuaga & L-4 & 30TWN376979 & 18 & 20 & 1 & Arenas, arcillas y limos \\
\hline & Izaixo & Moko & LI-1 & 30TWN405956 & 2,3 & 80 & 1,8 & Argilolitas \\
\hline & Urio & Atzueba & LU-1 & 30TWN411973 & 4 & 40 & 2,2 & Caliza recifal \\
\hline & Urio & Zulueta & LU-2 & 30TWN405988 & 6 & 20 & 1 & Calizas, areniscas y margas arcillosas \\
\hline & Lea & Oleta & L-5 & 30 TWN403993 & 22 & 20 & 1 & $\begin{array}{l}\text { Calizas y margas con argilolitas calcá- } \\
\text { reas }\end{array}$ \\
\hline \multirow{10}{*}{ ARTIBAI } & Artibal & Alcibar & A-1 & 30TWN379869 & 32 & 200 & 4 & Detritus \\
\hline & Axpc & Bolivar & AAx-1 & 30TWN372888 & 4 & 160 & 2 & Sustrato calcárco \\
\hline & Artibai & Alcibar & A-2 & $30 \mathrm{TWN} 386887$ & 5 & 140 & 2 & Sustrato calcáreo \\
\hline & Artibai & Marquina & A-3 & 30 TWN402907 & 8,5 & 80 & 1 & Arcillas, calizas y conglomerados \\
\hline & Urko & Barinaga & AU-1 & 30TWN414869 & 3 & 160 & 3,3 & $\begin{array}{l}\text { Basaltos, calizas, margas areniscosas, } \\
\text { arenas y arcillas }\end{array}$ \\
\hline & Echevaria & Marquina & AE-1 & 30 TWN43896 & 9 & 120 & 3 & Caliza recifal \\
\hline & Urko & Marquina & AU-2 & 30TWN413904 & 18 & 80 & 1 & $\begin{array}{l}\text { Arenisca, arcilla, caliza y conglomera- } \\
\text { do }\end{array}$ \\
\hline & Artibai & Uberuaga & A-4 & 30TWN413932 & 12 & 60 & 1 & Areniscas, arcillas y conglomerados \\
\hline & Amalloa & Kabizola & AA-I & 30TWN433933 & 4,5 & 80 & 1,6 & Caliza recifal \\
\hline & Artibai & Garro & A-5 & 30TWN443967 & 19 & 20 & 1 & Argilolitas \\
\hline \multirow[t]{2}{*}{ EGO } & Ego & Ermua & EGO-1 & $30 \mathrm{TWN} 403803$ & 2,5 & 200 & 2,8 & Areniscas, arcillas. arenas y calizas \\
\hline & Ego & Ermua & EGO-2 & 30TWN411811 & 4 & 160 & 2 & $\begin{array}{l}\text { Calizas, margas areniscosas, arenas y } \\
\text { arcillas }\end{array}$ \\
\hline
\end{tabular}

